\title{
Mindfulness for children with ADHD and Mindful Parenting (MindChamp): Protocol of a randomised controlled trial comparing a family Mindfulness-Based Intervention as an add-on to care-as-usual with care-as- usual only
}

Nienke M. Siebelink ${ }^{1,2^{*}}$ D, Susan M. Bögels ${ }^{3}$, Lisanne M. Boerboom², Noor de Waal ${ }^{2}$, Jan K. Buitelaar ${ }^{1,2}$, Anne E. Speckens ${ }^{4}$ and Corina U. Greven ${ }^{1,2,5}$

\begin{abstract}
Background: Self-control in childhood has been linked to long-term and cascading effects on health, academic, criminality, wealth and parenting outcomes. Hence it is important to target self-control deficits early in life. Selfcontrol deficits are a hallmark of Attention Deficit/Hyperactivity Disorder (ADHD). Even after receiving care-as-usual (CAU) for ADHD, impaired self-control often remains. Pharmacotherapy can be hampered by side-effects, low adherence and short-term effectiveness. Other limitations of CAU are decreased effectiveness when parents have ADHD and little effect on parental well-being. Mindfulness-Based Interventions (MBls) are an emerging nonpharmacological approach with potential to improve self-control and well-being in both children and parents. However, there is a lack of sufficiently powered randomised controlled trials (RCTs) to establish their effects in families with ADHD. This study protocol describes an RCT to investigate the effectiveness of a family MBI as an add-on to CAU in treatment of youth with ADHD, and is described in accordance with Standard Protocol Items: Recommendations for Interventional Trials (SPIRIT).
\end{abstract}

\footnotetext{
* Correspondence: n.siebelink@karakter.com

'Department of Cognitive Neuroscience, Donders Institute for Brain,

Cognition and Behaviour, Radboud University Medical Centre, Nijmegen, The Netherlands

${ }^{2}$ Karakter Child and Adolescent Psychiatry, University Center, Reinier Postlaan

12, Nijmegen 6525, GC, The Netherlands

Full list of author information is available at the end of the article
}

(c) The Author(s). 2018 Open Access This article is distributed under the terms of the Creative Commons Attribution 4.0 International License (http://creativecommons.org/licenses/by/4.0/), which permits unrestricted use, distribution, and reproduction in any medium, provided you give appropriate credit to the original author(s) and the source, provide a link to the Creative Commons license, and indicate if changes were made. The Creative Commons Public Domain Dedication waiver (http://creativecommons.org/publicdomain/zero/1.0/) applies to the data made available in this article, unless otherwise stated. 
(Continued from previous page)

Methods/design: An RCT will be conducted in N=100 children (aged 8-16 years) with ADHD and their parents. The experimental condition will consist of a family MBI (MYmind): 8-week group-based MBI for youth combined with parallel group-based Mindful Parenting for their parents, as an add-on to CAU. The control condition will consist of CAU-only. Assessments will take place at baseline, end of treatment (3 months later), 2 and 6 months' follow-up. Primary outcome measure will be an ecologically valid assessment of child self-control with the parent-rated Behaviour Rating Inventory of Executive Function (BRIEF). Secondary child outcome measures will be teacher-rated BRIEF, computerised self-control tasks and questionnaires on psychological symptoms (e.g. ADHD, symptoms of autism), well-being and mindfulness. For parental outcomes, secondary measures will be self-rated BRIEF, computerised self-control tasks and questionnaires on psychological symptoms, well-being and mindful parenting.

Discussion: The proposed RCT will take account of methodological limitations of previous studies on MBls in child ADHD populations. The current study will provide valuable information on family $\mathrm{MBI}$ as a potential effective intervention in targeting self-control deficits for youth with ADHD and their parents.

Trial registration: ClinicalTrials.gov NCT03220308. Retrospectively registered 18 July 2017.

Keywords: Self-control, Executive function, Attention, Adolescent, Parents, Mindfulness, ADHD,

\section{Background}

Self-control can be seen as an umbrella construct that bridges concepts from different disciplines (e.g., executive function, impulse control, attention-regulation, emotion-regulation, planning, delay of gratification, and cognitive flexibility) $[1,2]$. Poor self-control is an important feature of psychiatric disorders arising in childhood and adolescence, including Attention Deficit/ Hyperactivity Disorder (ADHD), oppositional defiant disorder (ODD), conduct disorder (CD), mood and anxiety disorders, substance use disorder, and gambling [3-5]. Early self-control predicts not only mental and physical health, but also current and later academic performance, wealth, criminality and parenting [1, 6-8]. For example, children with poor self-control at preschool age have been shown to be more likely to have substance dependence, financial troubles and a criminal record at age 32 [1]. Therefore, targeting self-control in interventions for youth with deficits in self-control appears of utmost importance.

Self-control can be trained, although it is unclear which interventions are most effective in improving self-control in youth [9]. Mindfulness-Based Interventions (MBIs) use exercises that train self-control capacities [10] and are increasingly gaining ground as an approach to increase self-control in youth $[11,12]$. Mindfulness is often defined as the trainable capacity to pay attention to experiences in the present moment, on purpose and without judgement [13, 14]. It has been suggested that MBIs enhance self-regulation through three interacting processes: enhanced attention control, improved emotional regulation, and altered self-awareness (diminished self-referential processing and enhanced body awareness) [15]. This is supported by an activation likelihood estimation meta-analysis of 21 structural neuroimaging studies in $\sim 300$ adult (mindfulness) meditation practitioners, showing that neural correlates of these cognitive processes are altered in meditators compared to non-meditators with a global medium effect size [16]. In addition, 78 functional neuroimaging studies were reviewed in an activation likelihood estimation meta-analysis with 527 healthy non-clinical adult meditation practitioners who actually meditated during scanning. The results indicate dissociable brain (de)activation patterns during different styles of meditation, which are congruent with the psychological and behavioural aims of each practice [17]. If neural mechanisms underlying self-control can be altered structurally and functionally by meditation practices, this likely results in effects on behavioural and cognitive measures of self-control as well.

Previous clinical trials show that self-control can be improved following MBIs in diverse populations. Randomised Controlled Trials (RCTs) show greater improvement after MBIs than active control conditions in executive functions of adults (undergraduates, $N=80$ ), adolescents (middle and high school students, $N=489$ ) and children (4-5 years, $N=60$ ) [18]. A meta-analysis on neuropsychological outcomes of MBIs in adult populations (clinical and non-clinical) found preliminary evidence for positive effects on general awareness and meta-awareness, cognitive flexibility and working memory [19]. However, evidence was weak for improvement of attentional control (alerting/sustained attention, orienting/selective attention and executive attention), executive function inhibition and mental set shifting. For youth (5-18 years old), a systematic review of interventions with a focus on yoga-, meditation-, and/or mindfulness-based techniques found significant effects of the interventions on attention and executive functioning with medium to large effect sizes in five of the thirteen included studies. However, study populations consisted of both non-clinical and clinical populations 
and methodological quality of the studies varied [20]. A meta-analysis of MBIs with youth found significantly higher effect sizes for clinical samples than for non-clinical samples [21]. In addition, an RCT found that specifically children with poor executive functioning show improvement in this area based on teacher and parent reports [22]. Hence, effectiveness of MBIs in increasing self-control may be greater in a more homogeneous clinical population with self-control difficulties.

As a consequence, MBIs are increasingly applied as an approach in the treatment of ADHD in which deficits in self-control are a central component [23]. ADHD is a common neurodevelopmental disorder characterised by impairing symptoms of inattention and hyperactivity-impulsivity, affecting $5-7.5 \%$ of all children worldwide [24]. The annual health care costs and societal 'costs of illness' of ADHD in youth are high and comparable in magnitude to other serious medical problems (e.g. stroke, asthma in children) and mental health problems (e.G. major depressive disorder) [25]. Furthermore, ADHD has a significant impact on the quality of life of the affected children comparable to other mental health conditions (e.g. anxiety disorders, autism spectrum disorders (ASD), mood disorders) and severe physical disorders (e.g. cerebral palsy, cancer) $[26,27]$. Moreover, quality of life of parents is also negatively affected by ADHD of their child, for example in terms of their psychological well-being, personal fulfilment, family and couple relationships and daily life activities [28].

Current care-as-usual (CAU) for children with ADHD consists of psychoeducation, pharmacotherapy and/or (cognitive-) behavioural treatments [29, 30]. Psychoeducation enhances parents' knowledge about ADHD and may enhance engagement in psychopharmacological treatment [31]. Pharmacotherapy can be effective in reducing core symptoms of ADHD and to a lesser extent improving other outcomes like quality of life, functioning [32], response inhibition, sustained attention and working memory [33]. However, pharmacotherapy can be hampered by side effects and low adherence [34-38]. Meta-analyses of (cognitive) behavioural interventions in the treatment of youth with ADHD show that these can improve parenting behaviour, increase parents' sense of self-worth and reduce child conduct problems. Specific modules may improve child social skills and academic functioning [31]. Nevertheless, this review also shows that behavioural treatment does not reduce observer rated ADHD symptoms of the child and no support was found for its effects on parental mental well-being [31, 39]. Moreover, cognitive training of attention and executive functioning in children with ADHD does not yield significant improvements in these areas [40]. Another limitation of both pharmacotherapy and behavioural interventions for ADHD is that treatment is less effective when parents have ADHD themselves [31, 41]. As ADHD runs in families and is highly heritable, this is often the case [42, 43]. In conclusion, CAU for ADHD is not sufficient for many families and a substantial subgroup of children with ADHD has remaining symptoms and impairment [44, 45]. MBIs for children and their parents are promising in targeting self-control, behavioural symptoms and parental outcomes [46-49].

Previous studies on MBIs as a treatment approach for youth with ADHD have been reviewed in several publications. A meta-analysis of studies on pre-post effects of MBIs on ADHD core symptoms in children and adults diagnosed with ADHD [50] yields an overall effect size of $d=-.66$ for reduction of inattention symptoms and $\mathrm{d}=-.53$ for reduction of hyperactivity/ impulsivity symptoms. In a subgroup analysis of the six included trials in children and adolescents only, medium effect sizes were calculated for both inattention $(\mathrm{d}=-.66)$ and hyperactivity/impulsivity $(\mathrm{d}=-.47)$. Next to effects on ADHD core symptoms, other clinical effects in children with an ADHD diagnosis ( $\leq 18$ years) and their parents were studied in a systematic review which included a broader range of interventions, i.e. studies with an intervention focusing on mindfulness and/or yoga techniques with either parents, children and/or parents plus children [51]. Eight of the sixteen studies targeted children only and another eight studies investigated a family-based intervention. Positive results were found for improvements after meditation-based interventions in parent-reports of child functioning, parent and/or teacher-reports of child internalising/ externalising behaviour, parental ADHD symptoms, parental satisfaction, parent-child relationships and parental happiness, but not in child self-reported happiness. Mixed or limited evidence was found for improvements in child ADHD symptoms, child self-esteem, child social functioning, child academic performance, child self-report of functioning, child-report of internalising/externalising symptoms and parental stress and over reactivity of the parent. Finally, larger effect sizes in child outcomes, lower incidence of poorer outcomes, and more favourable outcomes for parents resulted from trials with family-based interventions compared to child-only interventions. In conclusion, previous research on the effectiveness of MBIs in the treatment of youth with ADHD shows these are feasible interventions for ADHD with potential benefits across a broad range of outcomes including ADHD symptoms, well-being and outcomes for parents. However, the results above should be interpreted with caution due to limited methodological quality of the reviewed studies. It is of note that none of the systematic reviews on MBIs for youth with ADHD focused on self-control in particular. 
A few clinical trials looked at effects of MBIs on self-control as assessed with neurocognitive tests and/or with questionnaires in youth with ADHD. In a first RCT, comparing a family MBI with a waitlist control group in children with ADHD aged five to seven years $(N=100)$ and their parents, significantly greater improvement in the family MBI group compared to control group was found for child executive attention (conflict monitoring $)(\mathrm{d}=.41)$ [52]. This result is in line with a quasi-experimental trial on neurocognitive task performance following an MBI in adolescents $(N=8)$ and adults $(N=24)$ with ADHD, which also found significant improvements for measures of executive attention. In addition, significant effects were found for set-shifting [53]. A pilot pre-post-intervention study with a family MBI in children aged eight to twelve years $(n=11)$ found significant improvement with large effect sizes on objective attention tests, but not on parent ratings of self-control on the Behaviour Rating Inventory of Executive Function (BRIEF) [54]. In contrast, in a quasi-experimental trial with ten adolescents (aged 11-15 years) following a family MBI, self-control assessed with the BRIEF improved significantly at 8-weeks follow-up, but only according to father reports and not mother reports [55]. For youth with ADHD, no RCT has been published on effects of MBIs on ecologically valid questionnaire ratings of self-control (e.g. BRIEF). In adults with ADHD, a randomised waitlist controlled MBI trial $(N=20)$ resulted in significant group differences at the end of treatment favouring the MBI group on self-reported self-control (assessed in the laboratory and with ecological momentary assessment) and clinician rated self-control with large effect sizes. In contrast, no significant improvement was observed with the objective self-control tasks [56]. In another waitlist RCT on an MBI in adults with ADHD $(N=103)$, self-control as assessed with the BRIEF improved with a large effect size $(\mathrm{d}=.93)$ as well [57]. Furthermore, in a randomised CAU controlled MBI trial in 120 adults with ADHD, improvement on the BRIEF over time after MBI + CAU compared with CAU only was found, resulting in an effect size of $\mathrm{d}=.49$ at 6 -month follow-up [58]. In summary, implementing MBIs in the treatment of youth with ADHD to improve self-control is promising. Although there are preliminary positive results, the existing evidence in youth is insufficient due to a lack of studies with good methodological quality. RCTs with sufficient power are needed.

The current protocol describes an RCT comparing an 8-week family MBI for youth with ADHD and their parents (MYmind) in addition to CAU with continuation of CAU only. Participating children will have an ADHD diagnosis and comorbidities will be allowed (including ASD). The primary, secondary and tertiary aims correspond to the primary, secondary and tertiary measures that will be used in this study. The primary aim is to investigate the effectiveness of a family $\mathrm{MBI}$ in improving self-control of youth with ADHD, as assessed with ecologically valid parent-ratings (BRIEF-P, primary outcome). Different definitions are used in literature on self-control, some take a broad approach, other narrow, and opinions vary on what self-control comprises. We take a broad view on self-control in that it entails self-regulation of behaviour, emotion, cognition and attention. Given the moderate convergent validity of neurocognitive tests of self-control, we prefer ecologically-valid questionnaire ratings of self-control over neurocognitive tests [2]. The predictive validity of behavioural questionnaire ratings of self-control on clinically and societally relevant objective outcomes even decades later (e.g. months unemployed, single parenthood, criminal conviction) has been documented $[1,59]$. The secondary aim is to examine the effects of family MBI on child self-control as assessed with teacher-ratings (BRIEF-T) and objective computerised tasks and on psychological symptoms (e.g. ADHD symptoms, symptoms of ASD, brooding), well-being and mindfulness of the children (secondary outcome measures for children). In addition, we aim to examine effects of family MBI on parental self-control as assessed with ecologically valid self-ratings (BRIEF-A) and objective computerised tasks and on psychological symptoms, well-being and mindful parenting of the parents (secondary outcome measures for parents). Our tertiary aim is to look at the effects of family MBI on some exploratory measures such as mind-wandering. Finally, child saliva samples will be collected for (epi)genetic research and qualitative data will be collected to explore effects that are not captured with quantitative assessments and to explore facilitators and barriers of family MBI for youth with ADHD and their parents.

\section{Methods/design \\ Study design}

This is a parallel group RCT in which all participants with ADHD receive CAU. Randomisation will assign children with ADHD and their parents to either a family MBI (plus continuation of CAU) or to the control condition (continuation of CAU) with a 1:1 ratio.

\section{Participants}

The study population will consist of children aged 816 years with an ADHD diagnosis and at least one of their parents. The families will be recruited through the outpatient clinic of Karakter, an institute for child and adolescent psychiatry. Recruitment will take place at 
three different Karakter locations in the central eastern part of the Netherlands, namely Nijmegen, Arnhem and Ede (see also the clinical trial registration). In addition, families will be recruited by media advertisements.

\section{Eligibility criteria}

We will include families who meet the following criteria: a) child is $8-16$ years old and in the third grade of primary school or higher; b) child has a primary diagnosis of ADHD according to the Diagnostic and Statistical Manual of Mental Disorders (DSM-IV or DSM-5) system $[60,61]$ and confirmed by a structured interview (see Assessments section); c) child receives CAU for ADHD and has remaining ADHD symptoms (average score $>1.0$ on the investigator-rated DSM-5 items from the Conners' ADHD rating scale [62]); d) ADHD medication dose of child is stable, at least two weeks prior to baseline, or an informed decision is made on not taking ADHD medication; e) at least one parent is willing to participate. Exclusion criteria are a) psychosis, bipolar illness, active suicidality, untreated posttraumatic stress disorder or substance use disorder of the child that impedes functioning; b) psychosis, bipolar disorder, active suicidality, untreated posttraumatic stress disorder or substance use disorder of the parent that impedes functioning; c) child and/or participating parents have an estimated Intelligence Quotient (IQ) < 80; d) child and/or participating parents do not have adequate mastery of Dutch language; e) child and/or participating parents have participated in an $>=8$-week mindfulness programme in the past year or ever in a Mindful Parenting training; $f$ ) child or parents participate in another intervention study.

\section{Intervention}

\section{Family MBI (MYmind)}

We will use the MYmind course [63], a standardised, highly-structured protocol developed to target the specific difficulties that children with ADHD and their parents may confront when meditating. As self-control problems in regulating attention, impulses and motor activity are core issues of children with ADHD, the course is designed around these difficulties. The aim of MYmind is to teach children to meditate and to practice non-reactivity, that is, awareness of their impulses but not following them automatically. The course for the parents focuses on their role as a parent, through teaching them mindful parenting (e.g., to help guide their child with the meditations, practice non-reactive parenting and mindful communication with the child), but also directly addresses parents' own self-control and behaviour.

MYmind consists of eight weekly 90-min group sessions for children (groups of approximately 5-8), and parallel group sessions for their parents of equal length and duration. The child and parent sessions take place in separate rooms, with the exception of sessions 1,5 and 8 , where part of the session is together in the same room. In the sessions (playful) mindfulness exercises are done and education is given around the following themes, one per session: 1) Beginners' mind, 2) Home in our body, 3) The breath, 4) Distractors!, 5) Stress, 6) High way, walking way, 7) Acceptance \& autonomy, and 8) The future. Both children and parents receive daily homework during the eight weeks of training (15 min for child and 30-45 min for parent, 6 days/week). Theory and exercises are described in a workbook that both children and parents receive together with audio-files to guide the mindfulness exercises. As children with ADHD may have motivational problems, a reward system is incorporated in the course for the children to enhance motivation to practice in the sessions and at home. The 8-week course is followed by eight weeks of home self-practice, and a single joint child-parent 90-min booster session. In the booster session, participants share experiences of the mindfulness practice in the last eight weeks where they did not have the support of the group training, rehearse certain practices in order to remind the families of the possible joys and benefits of mindfulness practice, and are encouraged to think about how to implement mindfulness in their lives and to renew their intentions. Participants who attend four or more sessions will be considered as 'completers'.

The first trial on an MBI for children combined with Mindful Parenting was conducted by Bögels et al. (2008) [64], which resulted in the MYmind training. Since then, several non-randomised pilot studies have been conducted with the MYmind training in child and adolescent samples with ADHD [54, 55, 65-67] and with ASD $[68,69]$. Findings are promising and show that the MYmind training is feasible and acceptable [70].

\section{Mindfulness teachers}

Mindfulness teachers will have to be experienced and meet quality criteria for category 1 from the VMBN (Association of mindfulness-based teachers in the Netherlands and Flanders), which are in accordance with the 'UK Mindfulness-Based Teacher Trainer Network' [71]. The criteria for category 1 of the VMBN include a college or university degree; $>=$ three years of regular meditation experience; $>=$ one 10-day retreat or two 5-day retreats in the past four years; having participated in a Mindfulness-Based Stress Reduction (MBSR) [72] or Mindfulness-Based Cognitive Therapy (MBCT) [73] course; $>=150 \mathrm{~h}$ of education in MBSR/MBCT (skills training in giving formal and informal practices, theory and practice of psycho-education and inquiry, theoretical foundation, supervision, reflection report, adequate use of course 
materials and experience in giving training); $>=$ seven days in-service training per two years; giving $>=$ two MBSR/MBCT courses per two years. In addition, for the current study, mindfulness teachers will be trained thoroughly in the administration of the MYmind protocol by following a $5^{1 / 2}$-day advanced teacher training in MYmind given by its developer (SB). Following an advanced teacher training in MYmind also gives teachers access to the course materials (i.e. workbooks and audio files) for the participants. During the trial, the mindfulness teachers will be supervised by SB who has extensive expertise and experience working with the intervention delivered in this proposal. In the child-group there will be a mindfulness co-teacher, who will have experience with children with ADHD, in addition to the mindfulness teacher. All sessions during the 8-week training will be videotaped for treatment integrity purposes. Mindfulness teacher competency will be assessed on the Teaching Assessment Criteria (MBI:TAC; $[74,75])$ by experienced mindfulness teachers using a random selection of the videotapes on the Teaching Assessment Criteria (MBI:TAC [74, 75]).

\section{CAU}

According to the Dutch Multidisciplinary guidelines for the diagnosis and treatment of ADHD [76], CAU for children aged 8-16 years consists of psycho-education and the prescription of medication approved for ADHD and/ or evidence-based parent and/or teacher-administered behaviour therapy, preferably both medication and behaviour therapy. First-line option for medication is a psychostimulant, second-line options are atomoxetine or alpha-2 presynaptic agonists. Participants in the CAU-group will be informed that it is not allowed to participate in a mindfulness programme until the 2-month follow-up assessments of the study.

\section{Expectancy, satisfaction, compliance and healthcare consumption}

For family MBI and CAU condition, credibility and expectancy of clinical outcome will be evaluated with the parent-rated 6-item Credibility/Expectancy Questionnaire (CEQ, translation to Dutch by the authors for this study) [77]. The CEQ shows a good internal consistency and test-retest reliability [77]. Satisfaction with family $\mathrm{MBI} / \mathrm{CAU}$ will be assessed in parents and children at end of treatment, 2- and 6-month follow-up. Parental and child compliance with the mindfulness practices, and child adherence with any ADHD medication, will be assessed in the parents at the same three time points. Type and dose of possible ADHD-medication and other medication will be assessed at all time points for parent and child. At baseline parents will report which treatments for ADHD the child received prior to study participation. In addition, child healthcare consumption between assessments will be assessed with a 30-item adapted version of the parent-rated Trimbos and iMTA questionnaire on Costs associated with Psychiatric illness (TiC-P) [78] at end of treatment and both follow-up time points. Parental healthcare consumption during the whole study period will be assessed with the 46-item self-rated TiC-P at 6-month follow-up. The TiC-P is a valid questionnaire with a sufficient to high test-retest validity $($ ICC > .6) [79].

\section{Assessments \\ Descriptives}

Clinical ADHD diagnosis will be confirmed using a structured psychiatric interview (Schedule for Affective Disorders and Schizophrenia for School-Age Children-Present and Lifetime Version; K-SADS-PL) [80], adapted to DSM-5 and administered to a parent by a trained researcher. The focus of the interview will be on the behaviour of the child off medication in the past half year. Further, parents will be screened for the presence of adult psychiatric disorders, using the 10-item Kessler Psychological Distress Scale (K10) [81]. For those with elevated scores ( 25 or higher) a diagnostic interview (Mini-International Neuropsychiatric Interview; MINI) [82] will be administered to establish axis-I disorders by a trained researcher. In case no valid IQ test results of participants are available, full-scale IQ will be estimated by two subtests of the Wechsler Intelligence Scale for Children-Third Edition (WISC-III) [83] or Wechsler Adult Intelligence Scale-Third Edition (WAIS-III) [84]: Vocabulary and Block Design. These subtests together are known to correlate between $.88-.91$ with the Full-scale IQ $[85,86]$. Standardised questionnaires completed by parents as part of routine intake procedures at Karakter will give us a wide range of additional background information on variables such as age, gender, medical/medication history, ethnicity, family-structure and socioeconomic status. Some additional participant characteristics will be collected at baseline as there might be changes in family-structure or socioeconomic status between intake at Karakter and study participation. The Pubertal/Physical Development Scale (PDS) [87] will be used to assess the pubertal developmental stage of the child at baseline and 6-month follow-up.

\section{Primary outcome measure}

Parent-rated child self-control To measure self-control of the child, we will use the parent-rated version of the BRIEF [88], which assesses real-life self-control skills across situations and is used in clinical 
and research settings for ADHD. The questionnaire consists of 75 items with a 3-point rating scale ranging from 1 (never) to 3 (often) with higher scores indicating more problems in executive functioning. The BRIEF gives rise to a global executive composite score as well as two broad indexes (Behavioural Regulation and Metacognition) and eight subscales (Inhibit, Shift, Emotional Control, Initiate, Working Memory, Plan/Organise, Organisation of Materials, and Monitor). The internal consistency of the Dutch version is sufficient when administered to adolescents with ADHD (Cronbach's $\alpha$ coefficients ranging from .69 to .95) [55]. Test-retest reliability is high with intra-class correlations of $\geq .73$. The BRIEF demonstrates good convergent validity [88].

\section{Secondary outcome measures for children}

Teacher-rated child self-control Child self-control will also be assessed using teacher-ratings on the BRIEF-T [88]. Teachers have the advantage to be able to evaluate the child's behaviour in the context of their peers' behaviour and of the normative range, and are blinded to exactly which intervention is provided to the child at home and/or in the clinic [89]. The BRIEF-T consists of 75 items with the same 3-point rating scale and structure as the parent form. The Dutch version shows a good internal consistency, with Cronbach's $\alpha$ coefficients ranging from .88 to .98 , and a high test-retest reliability (ICC $=.77$ for the global executive composite score) [88].

Computerised tasks of child self-control Logan's Stop Signal task will be used to measure motor inhibition [90, 91]. In this task, participants are presented with go-trials which consist of the presentation of a stimulus (i.e. either the letter $\mathrm{O}$ or the letter $\mathrm{X}$ ) and are instructed to press the corresponding response button as quickly and accurately as possible. In $25 \%$ of the trials, at random, the go-trial is followed by an auditory stop-signal (i.e. a tone). In those trials, participants are required to withhold their response. The delay between the go and the stop signal is varied using a dynamic tracking algorithm, such that an average person has a $50 \%$ chance of correctly withholding their response at a stop-trial. The two main dependent behavioural measures will be the percentage of errors and the Stop Signal Reaction Time (SSRT), which is calculated by subtracting the Mean Delay from the Mean Correct Reaction Time [90]. Secondary behavioural outcome measures will be reaction time variability and speed accuracy trade off [92]. The Stop Signal task shows an adequate test-retest reliability when administered to children with ADHD (ICC = .72 for the SSRT) [91].

Temporal discounting refers to the decrease of subjective reward value as a function of increasing delay
[93]. In the Temporal Discounting task that will be used in this study [94, 95] participants are asked to choose between a smaller immediate reward (i.e. one, two, three of four eurocents) and a larger delayed reward (i.e. five eurocents) during forty trials. Choices are visually represented by two airplanes on a computer screen. Delays are represented by the "height" at which the planes are flying, and vary between five and sixty seconds. Participants choose the preferred plane which results in earning the chosen amount, immediately or after the appropriate delay. A maximum of two euros can be earned. The computer task will be followed by a debriefing on the child's choices and emotions regarding every delay interval using the Self-Assessment Manikin. This measure is shown to be a reliable, non-verbal method for assessing a person's emotional reaction in response to a certain event or stimulus [96]. Outcome measure will be the subjective reward value, defined as the magnitude of the smaller immediate reward that leads to indifference of the participant towards the larger delayed reward [93]. At this point, the participant shows no clear preference for one of the rewards, either the delayed or the immediate one. The real Temporal Discounting task (with actual money and delays) has a clear advantage compared to hypothetical tasks in terms of ecological validity [97] and is proven useful in assessing individual differences in children versus adolescents [94] and individuals with less and more hyperactivity/impulsivity symptoms [98].

The Probabilistic Reversal Learning task will be used to determine the cognitive ability to adapt behaviour according to changes in stimulus-reward contingencies, also known as cognitive flexibility [99]. An experimental study has shown impaired reversal learning to reflect reduced inhibitory control of affective responses, and therefore might be related to deficits in self-control [100]. In the Probabilistic Reversal Learning task participants repeatedly choose between two visual stimuli during eighty trials, followed by rewarding or punishing auditory feedback. In $80 \%$ of the trials, the choice is followed by actual feedback (i.e. rewarding tone with correct response and punishing tone with incorrect response) and in $20 \%$ by misleading feedback. The stimulus-outcome contingencies reverse unannounced after forty trials, resulting in the previous mostly rewarded stimulus now being mostly punished and vice versa. Subjects are instructed that the contingencies may change, but do not know when or how often. Thus, the difficulty in performing the task comes from the need to integrate feedback over a number of trials because negative feedback may either be a probabilistic punishment or signal a true reversal. Outcome measures will be Ratio Win-Stay (the number of times the participant repeats their choice following a rewarded trial, divided by 
the total times the participant repeats their choice), Ratio Lose-Shift (the number of times the participant shifts response following a punished trial, divided by the total times the participant shifts choices) and perseverative errors (two or more consecutive error responses during the reversal phase) [99]. The Probabilistic Reversal Learning task is a well-defined and ecological instrument which is widely used measuring cognitive flexibility in children [101] and adolescents [102]. Neurocognitive test instructions to children can be found in Additional file 1.

Child psychological symptoms Externalising and internalising problem behaviours of the child will be assessed through parent- and teacher-ratings on the Conners' Parent/Teacher Rating Scales-Revised: Long (CPRS-R:L [62]/CTRS-R:L [103]). We will use the subscales DSM-IV Inattentive Symptoms, DSM-IV Hyperactivity-Impulsive Symptoms, Oppositional, Anxious-Shy, Social problems and the Emotional Lability Index, resulting in questionnaires containing 44 (CPRS) and 37 items (CTRS). Items are scored on a 4-point rating scale ranging from 0 (not true at all) to 3 (very much true) with higher scores reflecting more symptoms. Both forms of the American version have a good internal consistency for all named subscales (Cronbach's $\alpha$ coefficient ranging from .80 to .94 ) except the Emotional Lability Index, which is sufficient (Cronbach's $\alpha$ coefficient ranging from .72 to .80) [103].

Symptoms of ASD will be assessed with the Social Responsiveness Scale (SRS) [104, 105] rated by parent and teacher. The 65 items can be organised into five subscales (i.e. Social Awareness, Social Cognition, Social Communication, Social Motivation, and Restricted Interests and Repetitive Behaviour) and two DSM-5 subscales (i.e. Social Communication and Interaction, and Restricted Interests and Repetitive Behaviour). Items are rated on a 4-point rating scale ranging from 1 (not true) to 4 (almost always true) with higher scores reflecting less social responsiveness. The Dutch version has a good internal consistency for both the parent- and teacher-form with Cronbach's $\alpha$ coefficients of .92 and higher [106].

Brooding will be assessed using the self-report subscale of the Ruminative Response Scale (RRS; items reformulated by the authors for children from age 8) $[107,108]$. Items are scored on a 4-point rating scale ranging from 1 (almost never) to 4 (almost always) with higher scores reflecting more brooding in response to sadness. The internal consistency of the American Brooding subscale is good $(\alpha=.80)$ when administered to adolescents [109].

Child well-being We will use parents report on their child's quality of life using the 11-item KIDSCREEN-10 [110]. Items are scored on a 5-point rating scale ranging from 1 (never) to 5 (always) with higher scores reflecting a higher health related quality of life. The internal consistency based on ten items is adequate $(\alpha=.78)$ [111].

Child mindfulness Mindfulness skills will be assessed using self-report on the Child and Adolescent Mindfulness Measure (CAMM) [112, 113]. The CAMM consists of ten items that are scored on a 5-point rating scale ranging from 0 (never true) to 4 (always true) with lower scores reflecting better mindfulness skills. Internal consistency for the Dutch version was reasonable when administered to children $(\alpha=.71)$ and good for adolescents $(\alpha=.80)$ [112].

\section{Secondary outcome measures for parents}

Self-rated parental self-control Adult versions of the self-control measures for children will be administered to parents. The BRIEF-A [114] is a 75-item self-report questionnaire comparable to the child version in rating scale and structure, but with slightly different subscales (i.e. Inhibit, Shift, Emotional Control, Self-Monitor, Initiate, Working Memory, Plan/Organise, Task Monitor and Organisation of Materials). The Dutch version shows a good internal consistency for the global executive composite score and both indexes (Cronbach's $\alpha$ coefficient ranging from .92 to .96) and sufficient internal consistency for all subscales [114].

Computerised tasks of parental self-control The parents' version of the Stop Signal task and the Probabilistic Reversal Learning task will be identical to those described for children. The Stop Signal task shows an adequate test-retest reliability when administered to adults without a DSM-IV diagnosis in the past year $(r=.65$ for the SSRT) [115]. The Probabilistic Reversal Learning task shows adequate results when administered to adults with schizophrenia (ICC ranging from .49 to .68) [116].

As the Temporal Discounting task that will be used for children is not appropriate for use in adults, a hypothetical Temporal Discounting task will be administered to the parents. Participants make a series of choices regarding fictional amounts of money (rewards), ranging between one and hundred euros, available now versus after a specified delay interval (i.e. one month, one year, five years, ten years). Instructions are presented on the screen and are identical to those used by R. M. Hurst, H. O. Kepley, M. K. McCalla and M. K. Livermore [117]. For each trial, two variable rewards are presented in a random order, to increase the likelihood that the decisions are based on the amount in the individual trial and not on the previous trial. The participant's score on the different delay intervals is the reward value 
where they switch between taking the delayed reward to taking the immediate one. The hypothetically Temporal Discounting task is widely used in various studies [97] and shows good internal consistency $(\alpha=.89)$ when administered to adults with and without self-reported ADHD [117]. Neurocognitive test instructions to parents can be found in Additional file 1.

Parental psychological symptoms Symptoms of ADHD will be assessed using self-report on the Dutch version of the ADHD DSM-IV rating scale [118], which consists of 26 items concerning behaviour in the last two weeks (23 items) and childhood (three items). The latter three items are useful for estimating ADHD diagnosis, as onset of ADHD symptoms needs to be before the age of twelve according to the DSM-5. Items are scored on a 4-point rating scale ranging from 0 (never or seldom) to 3 (very often) with higher scores indicating more ADHD traits. The internal consistency is high for the Inattentiveness dimension $(\alpha=.83)$ and adequate for both the Hyperactivity $(\alpha=.75)$ and Impulsivity dimension $(\alpha=.72)$ [118].

Symptoms of ASD will be assessed using the self-rated 10-item Short Autism-Spectrum Quotient (AQ-10) [119, 120]. Each item is rated on a 4-point rating scale $(1=$ definitely agree, $2=$ slightly agree, $3=$ slightly disagree, 4 = definitely disagree) with higher scores indicating more symptoms of ASD. The internal consistency is good for the English 10-item version $(\alpha>$.85) [120].

Symptoms of depression, anxiety and stress will be assessed using the self-rated 21-item Depression Anxiety Stress Scale (DASS-21) [121, 122] rated on a 4-point rating scale ranging from 0 (did not apply to me at all) to 3 (applied to me very much of most of the time). Higher scores reflect more symptoms of depression, anxiety and/or stress. All subscales of the Dutch version show a good internal consistency with Cronbach's $\alpha$ coefficient ranging from .85 to .94 for students and a clinical sample of adults with an anxiety disorder and/or depression [122].

Brooding will be assessed using the Brooding subscale of the self-rated RRS $[107,108]$ containing five items that are rated on a 4-point rating scale ranging from 1 (almost never) to 4 (almost always). Higher scores indicate more brooding in response to sadness. The internal consistency of the Dutch Brooding subscale is sufficient $(\alpha=.78)$ when administered to adults [108].

Parental well-being Quality of life will be assessed using the self-rated 5-item World Health Organization-Five Well-Being Index (WHO-5) [123]. Each item is scored on a 6-point rating scale ranging from 5 (all the time) to 0 (not at all). Higher scores reflect higher psychological well-being. The WHO-5 shows an adequate validity as an outcome measure in clinical controlled trials, sensitive for assessing change [124].

Emotional, psychological and social well-being will be assessed using self-report on the 14-item Mental Health Continuum-Short Form (MHC-SF) [125]. Each item is scored on a 6-point rating scale ranging from 0 (never) to 5 (every day) with higher scores indicating more positive mental health. The Dutch version shows high internal consistency for the total MHC-SF score $(\alpha=.89)$ and the two subscales Emotional and Psychological Well-Being (both $\alpha=.83$ ), and adequate for the third subscale Social Well-Being $(\alpha=.74)$ [125].

Mindful parenting We will use self-ratings on the 31-item Interpersonal Mindfulness in Parenting scale (IM-P) [126, 127] assessing 1) Listening with Full Attention; 2) Compassion for the Child; 3) Non-judgmental Acceptance of Parental Functioning; 4) Emotional Nonreactivity in Parenting; 5) Emotional Awareness of the Child and 6) Emotional Awareness of Self. Items are scored on a 5 -point rating scale $(1=$ never true, $2=$ rarely true, $3=$ sometimes true, $4=$ often true, $5=$ always true) with higher scores reflecting more mindful parenting. The Dutch version of the IM-P shows a good internal consistency based on 29 items $(\alpha=.89)$ [126].

\section{Tertiary measures for children}

Self-rated We will use the 8-item subscale Body Awareness of the Body Experience Questionnaire for Children (BEQC) as a tertiary measure, which shows an adequate internal consistency for the Dutch version $(\alpha=.71)$ [128]. Sensory-processing sensitivity will be assessed using the 12-item Highly Sensitive Child Scale (HSCS). The HSCS shows an adequate internal consistency $(\alpha$ $=.79$ ) [129]. For children aged eleven and older we will use the 24-item Inventory of Callous-Unemotional Traits (ICU) assessing uncaring, callousness and unemotional traits. The Dutch version shows a good internal consistency $(\alpha=.89)$ [130]. Self-compassion will be assessed by using one item per subscale of the Self-Compassion Scale [131] with the highest factor loading [132]. This resulted in six exploratory items which were reformulated by the authors for children. Mind-wandering will be assessed using an exploratory questionnaire (items developed by JB for this study, 2015) containing three items (e.g. "When you are busy with schoolwork, a task or a chore, do you notice that you are daydreaming?").

Parent-rated The 18-item DSM-IV-based Strengths and Weakness of ADHD Symptoms and Normal Behaviour 
(SWAN) [133] will be used to assess ADHD traits (inattentiveness and hyperactivity-impulsivity). The Dutch version of the SWAN shows a good internal consistency $(\alpha>.87)$ when administered to parents $(\alpha=.88)$ [134] and will be included as it provides good resolution across the full range of continuous ADHD traits, thereby potentially providing additional information to the Conners' Rating Scales [135]. Neuroticism, as a covariate for the HSCS, will be assessed by administering the 5-item Big Five questionnaire [136]. Sleeping habits will be assessed using the 5-item Sleep Questionnaire used in standard clinical care assessing problems with sleeping in, sleeping through and total amount of sleep compared to children of the same age.

Teacher-rated The above described 18-item SWAN [133] will be administered to teachers, also showing a high internal consistency for the Dutch version $(\alpha=.91)$ [134].

Tasks Reading speed and accuracy will be assessed with the Dutch One Minute Reading Test; a test of word decoding, in which children are instructed to read as many words from a list as possible within one minute. The test shows a good test-retest reliability with high correlations ranging from .80 to .92 [137].

\section{Tertiary measures for parents}

Self-rated The 12-item Highly Sensitive Person Scale (HSPS) will be administered to parents assessing sensory-processing sensitivity, showing a good internal consistency $(\alpha=.89)$ when administered to adults [136]. Neuroticism, as a covariate for the HSPS, will be assessed by administering the 5-item Big Five questionnaire [136]. For the six subscales of the Self-Compassion Scale, the item that correlates the most with the total scale will be selected to create a 6-item questionnaire to explore self-compassion [132].

\section{Qualitative research}

Qualitative interviews with families and mindfulness teachers will be conducted after the family MBI, with the aim to capture the richness and heterogeneity of experiences of families with ADHD potentially not tapped by the quantitative assessments, and to facilitate adaptations to the MYmind protocol based on participant and mindfulness teacher experience. Purposive sampling will be used to include a subset of families with different backgrounds, age groups, gender and drop-outs will also be interviewed. Data will be generated until saturation is reached. The interviews are semi-structured using a topic guide focusing on two main topics: 1) facilitators and barriers to participating in family MBI training; and 2) effects of family MBI on parent, child and parent-child interactions. Interviews will be conducted separately with parent, child and mindfulness teachers. All interviews will be audio recorded, transcribed verbatim and analysed in the Atlas.ti software using Grounded Theory [138]. Analysis will be performed by a team of researchers to ensure no data get lost. Essential aspects of qualitative research concerning relevant participant selection, appropriate data collection methods, comprehensive data collection process and data analysis will be followed [139].

\section{Biological assessments}

Studying (epi)genetic factors alongside environmental factors in relation to family MBI can help understand the biological basis of family MBI. Five saliva samples will be drawn from children for DNA and RNA isolation and assessment of biomarkers: one OG-500 DNA at baseline for genotyping of DNA; one Oragene OG-575 DNA at baseline and one at end of treatment for methylomic profiling of CpG DNA sequences; and one Oragene RE-100 RNA at baseline and one at end of treatment for profiling of transcriptomics (mRNA), microRNA expression and epigenomic profiling. The collection of saliva during end of treatment will be scheduled during the same time of the day as the collection at baseline within participants to account for hormonal fluctuations. Children will be asked to refrain from food and drinks (except water) and cigarettes thirty minutes before collection. Samples will be processed under barcode in a validated and well-controlled pipeline designed to process samples for clinical use. The material of biological nature will be stored at -20 or $-80{ }^{\circ} \mathrm{C}$ (as appropriate; RE-100 RNA vials to be stored at $-80^{\circ}$ $\mathrm{C}$ to minimise any RNA degradation). Data will be stored in an automated, validated laboratory information system (Labvantage: www.labvantage.com/).

\section{Procedure and treatment allocation}

Recruitment will take place within the outpatient clinics of Karakter through screening by the researchers of currently treated patients or through referral by the psychiatrist. In addition, recruitment will take place via media advertisement (e.g. flyers and website). In case there are no contra-indications (i.e. exclusion criteria, crisis situation or family does not want to be approached for scientific research), families will be contacted by phone to inform them about the study and to establish whether they meet the inclusion criteria. Next, information letters will be sent to parent and child via e-mail. Families will have a minimum of two weeks to consider participation. Families willing to participate will then be sent forms of informed consent in the post: parental consent of child participation, parental consent of own participation, child consent (age $>=12$ years) of own 
Table 1 Assessments for children and time points

\begin{tabular}{|c|c|c|c|}
\hline \multirow{2}{*}{$\frac{\text { Assessments for children }}{\text { Descriptives }}$} & \multicolumn{3}{|c|}{ Time points } \\
\hline & & & \\
\hline Demographics & T0 & & \\
\hline WISC-III (Vocabulary and Block Design) & T0 & & \\
\hline Investigator-rated K-SADS-PL & T0 & & \\
\hline Self-rated PDS & T0 & & T3 \\
\hline \multicolumn{4}{|l|}{ Primary outcome measure } \\
\hline \multicolumn{4}{|l|}{ Parent-rated child self-control } \\
\hline BRIEF-P & T0 & $\mathrm{T} 1 \mathrm{~T} 2$ & T3 \\
\hline \multicolumn{4}{|l|}{ Secondary outcome measures } \\
\hline \multicolumn{4}{|l|}{ Teacher-rated self-control } \\
\hline BRIEF-T & T0 & $\mathrm{T} 1 \mathrm{~T} 2$ & \\
\hline Computerised tasks of self-control & T0 & $\mathrm{T} 1$ & T3 \\
\hline \multicolumn{4}{|l|}{ Psychological symptoms } \\
\hline $\begin{array}{l}\text { Parent-rated CPRS-L:R (subscales DSM-IV inattentive } \\
\text { symptoms, DSM-IV hyperactivity-impulsive symptoms, } \\
\text { oppositional, anxious-shy, social problems, emotional } \\
\text { lability index) }\end{array}$ & TO & $\mathrm{T} 1 \mathrm{~T} 2$ & $\mathrm{~T} 3$ \\
\hline $\begin{array}{l}\text { Teacher-rated CTRS-L:R (subscales DSM-IV inattentive } \\
\text { symptoms, DSM-IV hyperactivity-impulsive symptoms, } \\
\text { oppositional, anxious-shy, social problems, emotional } \\
\text { lability index) }\end{array}$ & TO & T1 T2 & \\
\hline Parent-rated SRS & T0 & $\mathrm{T} 1$ & T3 \\
\hline Teacher-rated SRS & T0 & $\mathrm{T} 1$ & \\
\hline Self-rated RRS (Brooding) & T0 & $\mathrm{T} 1$ & T3 \\
\hline \multicolumn{4}{|l|}{ Well-being } \\
\hline Parent-rated KIDSCREEN-11 & T0 & $\mathrm{T} 1$ & T3 \\
\hline \multicolumn{4}{|l|}{ Mindfulness } \\
\hline Self-rated CAMM & T0 & $\begin{array}{lll}\mathrm{T} 1 & \mathrm{~T} 2 & \mathrm{~T}\end{array}$ & T3 \\
\hline \multicolumn{4}{|l|}{ Tertiary measures } \\
\hline \multicolumn{4}{|l|}{ Self-rated } \\
\hline $\mathrm{BEQC}$ & T0 & $\mathrm{T} 1$ & T3 \\
\hline $\mathrm{HSCP}$ & T0 & & \\
\hline ICU (ages 11+) & T0 & $\mathrm{T} 1$ & \\
\hline Self-compassion (exploratory items) & TO & $\mathrm{T} 1$ & T3 \\
\hline Mind-wandering (exploratory items) & T0 & $\begin{array}{lll}\text { T1 } & \text { T2 } & 7\end{array}$ & T3 \\
\hline \multicolumn{4}{|l|}{ Parent-rated } \\
\hline SWAN (optional) & T0 & T1 & T3 \\
\hline Neuroticism (covariate for HSCP) & T0 & & \\
\hline Sleeping habits & T0 & T1 & T3 \\
\hline \multicolumn{4}{|l|}{ Teacher-rated } \\
\hline SWAN & T0 & T1 & \\
\hline Tasks & & & \\
\hline
\end{tabular}

Table 1 Assessments for children and time points (Continued)

\begin{tabular}{lc}
\hline Assessments for children & Time points \\
\hline One Minute Reading Test & T0 T1 T0 T1 \\
Saliva collection & T3 \\
\hline T0 baseline, T1 end of treatment, T2 2-month follow-up, and T3 6-month follow-up, \\
WISC-III Wechsler Child Intelligence Scale-Third Edition, K-SADS-P Schedule for \\
Affective Disorders and Schizophrenia for School-Age Children-Present and Lifetime \\
Version, PDS Pubertal Development Scale, BRIEF-P Behaviour Rating Inventory of \\
Executive Function-Parent form, BRIEF-T Behaviour Rating Inventory of Executive \\
Function-Teacher form, CPRS-L:R Conners' Parent Rating Scales-Revised: Long, CTRS- \\
L:R Conners' Teacher Rating Scales-Revised: Long, SRS=Social Responsiveness Scale, \\
RRS= Ruminative Response Scale, CAMM = Child and Adolescent Mindfulness \\
Measure, BEQC=Body Experience Questionnaire for Children, HSCP=Highly Sensitive \\
Child Scale, ICU=Inventory of \\
Callous-Unemotional Traits, and SWAN=Strengths and Weakness of ADHD \\
Symptoms and Normal Behaviour
\end{tabular}

participation. Participants not referred by Karakter will also give consent to request medical data from the institute/clinician who diagnosed ADHD. The participants will be randomised after completion of the informed consent forms. In case families withdraw consent before the baseline assessments, randomisation will be undone and participants can be replaced, in order not to let non-participants imbalance the treatment groups for the primary analyses. School teachers and general practitioners of included participants will be informed about the study via telephone, email and/or postal letter. The school teachers will be requested not to ask the family about treatment allocation to keep them 'blinded'. Likewise, the participants will be requested not to speak about treatment allocation to the school teachers.

Assessments will take place at baseline (T0), end of treatment (T1), two (T2) and six (T3) months after end of treatment. For the family MBI condition, T0 will be scheduled in the two weeks prior to the start of the intervention and $\mathrm{T} 1$ in the two weeks after the 8th session of the MYmind training. The booster session will be followed by the first follow-up assessments (T2) which will be two months after T1 and the second follow-up assessments (T3) will be scheduled half a year after T1. Intervals between assessments will be kept similar for the family MBI and CAU condition. For baseline, end of treatment and 6-month follow-up assessments, participants will be invited to a location of Karakter. Assessments will be conducted by a researcher or research assistant. Participants will be asked to interrupt ADHD medication intake $48 \mathrm{~h}$ prior to these assessments. For the 2-month follow-up assessments, both parent and child will receive an invitation to complete questionnaires online (not at Karakter). School teachers will be asked to complete all questionnaires online at baseline, end of treatment and 2-month follow-up. See Table 1 for which assessments for children will be taken at which time points and Table 2 for assessments for parents and time points. Further, see Fig. 1 for a flowchart on recruitment and the study procedure. 
Table 2 Assessments for parents and time points

\begin{tabular}{|c|c|c|c|c|}
\hline Assessments for parents & \multicolumn{4}{|c|}{ Time points } \\
\hline \multicolumn{5}{|l|}{ Descriptives } \\
\hline Demographics & \multicolumn{4}{|l|}{ TO } \\
\hline WAIS-III (Vocabulary and Block Design) & \multicolumn{4}{|l|}{ T0 } \\
\hline Self-rated K10 & \multicolumn{4}{|l|}{ TO } \\
\hline Investigator-rated MINI & \multicolumn{4}{|l|}{ T0 } \\
\hline \multicolumn{5}{|l|}{ Secondary outcome measures } \\
\hline \multicolumn{5}{|l|}{ Self-rated self-control } \\
\hline BRIEF-A & T0 & $\mathrm{T} 1$ & $\mathrm{~T} 2$ & $\mathrm{~T} 3$ \\
\hline Computerised tasks of self-control & T0 & $\mathrm{T} 1$ & & $\mathrm{~T} 3$ \\
\hline \multicolumn{5}{|l|}{ Psychological symptoms } \\
\hline Self-rated ADHD DSM-IV rating scale & T0 & $\mathrm{T} 1$ & $\mathrm{~T} 2$ & $\mathrm{~T} 3$ \\
\hline Self-rated AQ-10 & T0 & $\mathrm{T} 1$ & & $\mathrm{~T} 3$ \\
\hline Self-rated DASS-21 & T0 & $\mathrm{T} 1$ & & $\mathrm{~T} 3$ \\
\hline Self-rated RRS (Brooding) & T0 & $\mathrm{T} 1$ & & $\mathrm{~T} 3$ \\
\hline \multicolumn{5}{|l|}{ Well-being } \\
\hline Self-rated WHO-5 & T0 & $\mathrm{T} 1$ & & $\mathrm{~T} 3$ \\
\hline Self-rated MHC-SF & T0 & $\mathrm{T} 1$ & & $\mathrm{~T} 3$ \\
\hline \multicolumn{5}{|l|}{ Mindful parenting } \\
\hline Self-rated IM-P & T0 & $\mathrm{T} 1$ & $\mathrm{~T} 2$ & $\mathrm{~T} 3$ \\
\hline \multicolumn{5}{|l|}{ Tertiary measures } \\
\hline Self-rated HSPS & T0 & & & \\
\hline Self-rated neuroticism (covariate for HSPS) & T0 & & & \\
\hline Self-rated self-compassion (exploratory items) & TO & T1 & & T3 \\
\hline
\end{tabular}

Note. T0 baseline, T1 end of treatment, T2 2-month follow-up, and T3 6-month follow-up, WAIS-III=Wechsler Adult Intelligence Scale-Third Edition, $\mathrm{K} 10=10$-item Kessler Psychological Distress Scale, MINI = Mini-International Neuropsychiatric Interview, BRIEF-A = Behaviour Rating Inventory of Executive Function-Adult version, $\mathrm{AQ}-10=10$-item Short Autism-Spectrum Quotient, $\mathrm{RRS}=$ Ruminative Response Scale, WHO-5 $=5$-item World Health Organization-Five Well-Being Index, MHC-SF = Mental Health Continuum-Short Form, DASS-21 = 21item Depression Anxiety Stress Scale, IM-P=Interpersonal Mindfulness in Parenting Scale, and HSPS=Highly Sensitive Person Scale

\section{Sample size \\ Power calculation}

Power analyses are based on the primary hypothesis that family MBI, relative to control, will lead to improvement in self-control (as measured by the BRIEF) at end of treatment. The BRIEF has shown modest to large effect sizes in MBI studies for clinical samples, including bipolar disorder [140][Cohen's d effect sizes from 0.08-1.33 on self-report BRIEF] and adult ADHD [57][Cohen's d effect size from 0.43 to 0.93 on self-report BRIEF]; [56][Cohen's d effect size from 0.67 to 1.72 on self-report BRIEF]. Further, in previous work using the MYmind programme (unpublished data on a larger non-randomised waitlist-controlled pilot in youth with ADHD $N=195$ families using parent-report BRIEF; see also [55][Cohen's d effect size from 0.0 to 1.8 on parent-report BRIEF; 0.2 to 0.5 on teacher-rated BRIEF]), a moderate effect on the BRIEF was found. Therefore power calculations were based on a moderate effect size of 0.4. Power analyses were run using the software G*power 3.1 and based on the procedure by G. F. Borm, J. Fransen and W. A. Lemmens [141]. Using this method, the $\mathrm{N}$ per group is obtained from the $\mathrm{N}$ per group for t-test multiplied by the design factor (D). The design factor $D=1-r^{2}$, where $r^{2}$ is the correlation between baseline and end of treatment on the BRIEF. We determined $\mathrm{r}$ using the data of a normative sample over a comparable time frame (six weeks): .86 [142]. As the period between baseline and end of treatment is slightly longer in this trial (around eleven weeks) the power analyses was conducted using a more conservative estimate of $r=.76$, hence $\mathrm{D}=1-.76^{2}=.42$.

Based on a power of $80 \%$, a two-tailed test with $\alpha=.05$ and an estimated effect size of 0.4 , we need $100^{*} .42=42$ children in each group. Past studies conducted using the MYmind programme (see section Intervention) using non-randomised designs in youth with ADHD and ASD found attrition rates from 5 to $15 \%$. Therefore, we will recruit a slightly larger sample $(2 \mathrm{xN}=50$, total $N=100)$, allowing room for attrition of around $15 \%$.

\section{Randomisation}

Randomisation by means of minimisation [143] will be performed after completion of the informed consent forms. The treatment group that most strongly minimises the imbalance between the two conditions is chosen to allocate the patient. To balance possibly prognostic factors across treatment groups, the following procedure will be performed: first, a stratification for age group is performed (elementary school or secondary school). Then, block randomisation is performed (with varying predefined block sizes), combined with minimisation. The three minimisation factors are: 1) medication for ADHD at baseline (medication versus no medication); 2) gender (male versus female); and 3) age (younger (child: age $8-10$ or adolescent: age 12-14) versus older (child: age 10-12 or adolescent: age 1416)). Minimisation factors are of equal importance, and block randomisation always wins over minimisation. Concealment of allocation is guaranteed as the sequence behind the randomisation will be unknown by the executing researcher.

\section{Data collection, management analysis}

As soon as the participant is enrolled, across all assessments he or she will only be identifiable via a unique pseudocode identifier to anonymise all data. A separate protected data-base will link the unique pseudocode to the participants' names. Anonymous and non-anonymous (e.g. informed consent forms) data 


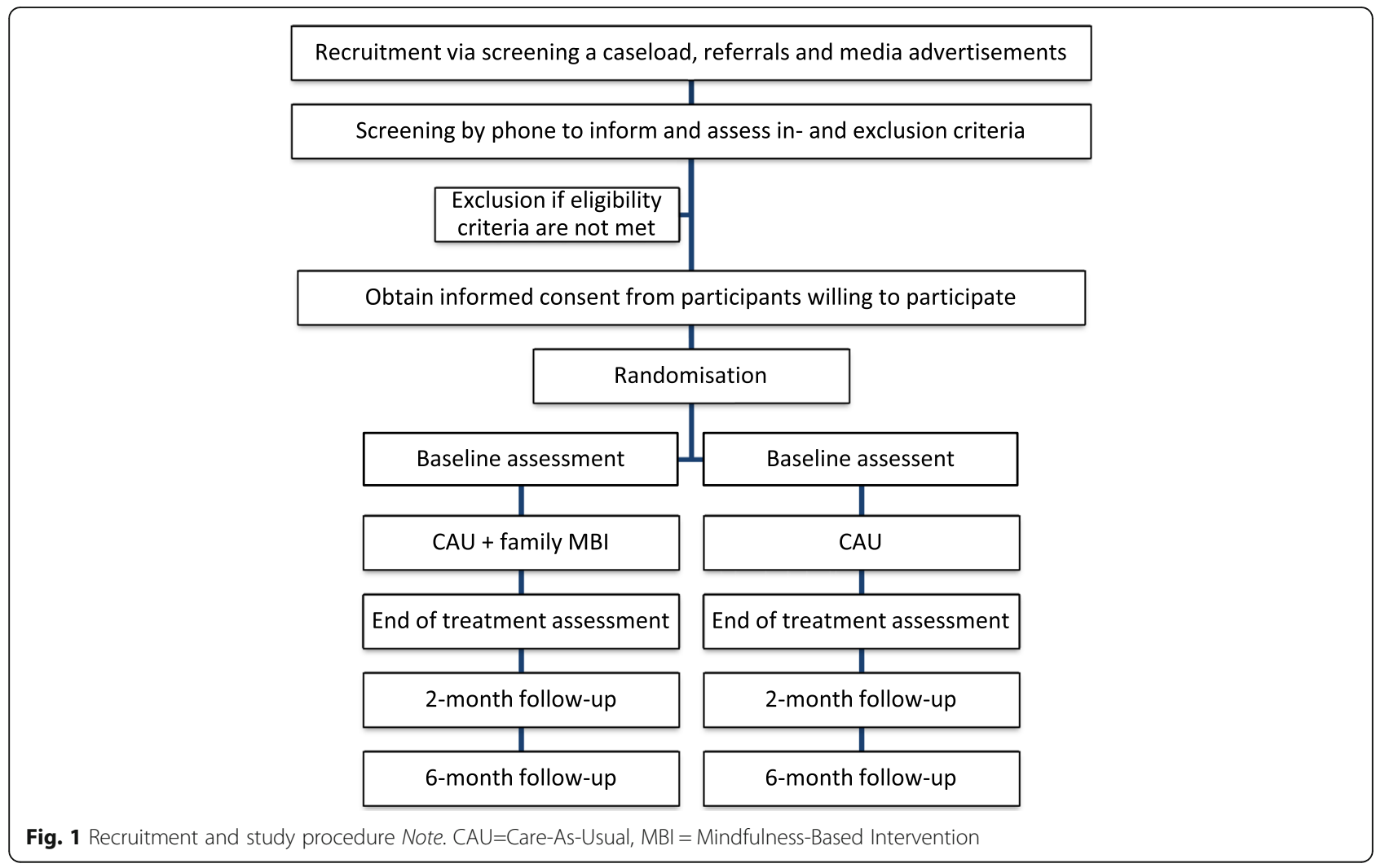

will be stored in separate password protected folders. Video-data of the sessions will be transported with an encrypted external hard disk and stored on a network attached storage only accessible for the appointed researchers. Questionnaire data will be collected and stored with the online electronic data capture software CASTOR EDC [144], which tracks and logs any manual changes made to raw data and is fully Good Clinical Practice (GCP) compliant. Quantitative data that is first collected on paper (e.g. IQ test, diagnostic interview) will be manually entered in CASTOR EDC and the entry will be double checked by another researcher. To prevent missing data, the force step completion function will be used so informants can only continue with the questionnaire when all items are completed. When questionnaires are not completed within the expected period, participants/school teachers will be sent online reminders. If no response follows they will be contacted per telephone to discuss the absence of response and to motivate them to complete the questionnaires, if necessary with concessions (e.g. fewer questionnaires). In case participation is discontinued, reasons will be noted and participants will be asked to complete a drop-out questionnaire with the main outcome measures (BRIEF-P, BRIEF-A, CPRS and parental ADHD self-report). Clinically informative results derived from child- and parent-rated questionnaire data will be reported by the researchers and sent to the involved participant after study participation ends.

\section{Statistical analyses}

Data will be analysed and reported according to CONSORT guidelines. Baseline demographic and clinical characteristics of the family MBI- and CAU-groups will be compared to examine whether these have been distributed evenly across the two groups by the randomisation. The primary analyses will be aimed at comparing results on the outcome measures at end of treatment between the family MBI and CAU condition, controlling for baseline levels and possible other baseline differences between intervention and CAU groups. To test our primary hypothesis that assignment to family MBI (relative to CAU) will improve child self-control, we will use ANCOVA with group condition (family MBI versus $C A U$ ) as the primary independent variable, and child self-control (continuous BRIEF-P score) at end of treatment as the primary dependent variable and child self-control at baseline as covariate. Cohen's d effect sizes will be calculated. Analyses will be conducted both according to intention-to-treat principle, and per-protocol on completers (family MBI condition: child and parent attended $>=4$ family MBI sessions). Intention-to-treat analyses will be conducted 
using available case analysis (not considering individuals with missing data) as well as imputation, as described in [145]. In case both parents participate in the assessments, data of the parent with the most complete dataset will be used for the ANCOVA, and if both are complete, the sex of the parent (or age, for same-sex parents) will determine which dataset will be used so that the fathers:mothers ratio is most similar across both study arms.

Secondary analyses, aimed at examining the consolidation of treatment effects at follow-ups, will use multilevel modelling with time point as repeated measurement, following the approach described in [58]. Moreover, we will conduct a set of complementary analyses to derive prediction models for response to intervention from baseline characteristics, and explore interactions between child and parental outcomes.

\section{Monitoring}

A data protection officer independent from the researcher will be assigned and monitors 1) the protection of the rights and well-being of the participants; 2) whether the reported research data is accurate and completely verifiable in source documents; and 3) whether the implementation of the research is consistent with the approved protocol/amendment(s) at that time, with GCP and the applicable legal requirements [146]. After including the first five participants, all data will be monitored. That is, the accuracy and completeness of all data (on item/trial level) per participant will be checked. After including participant five to ten, the completeness and accuracy of the data on summary/scale level will be checked for all participants. Thereafter, data on summary/scale level will be checked randomly for one in five participants. Outcomes of this data monitoring will be summarised in an overview and reported to all research personnel.

Based on previous studies on MBI for children and Mindful Parenting, deterioration as a result of family MBI is not expected. Therefore, no interim analysis will be conducted. Adverse events (AEs) are defined as any undesirable experience occurring to a participant during the study, whether or not considered related to the family MBI. All AEs reported spontaneously by the participant or observed by the researcher or mindfulness teachers will be recorded. All serious adverse events (SAEs, resulting in death, life threatening, requiring hospitalisation or other important medical events) will be communicated to the principal and coordinating researchers, who will report the SAEs to the accredited ethics committee that approved the protocol, within fifteen days after the sponsor has first knowledge of the SAE. SAEs that result in death or are life threatening should be reported expedited. The expedited reporting will occur not later than seven days after the responsible researcher has first knowledge of the SAE.

\section{Discussion}

Self-control is a malleable determinant of success in health, wealth, parenting, and avoiding crime [147]. Hence, improving self-control in children with self-control deficits has an important impact on their life and society. An example of a clinical population in which targeting self-control in treatment is pressing is youth with ADHD. ADHD is associated with adverse outcomes including impediment on academic achievement, mental and substance use disorders, criminality, and employment [148]. Current CAU for youth with ADHD is often not sufficient in improving self-control. Furthermore, CAU is generally not focused on mental health and well-being of the parents, although this has impact on the (treatment of the) child as well. ADHD medication can have undesired side effects, is refused by some families, compliance may be low, and improvements do not last after medication discontinuation [35]. These shortcomings might be addressed by offering a family-based MBI in addition to CAU. Self-regulation is present at the basis of MBIs and work by cognitive neuroscientists demonstrates that brain structures and functions that are involved in self-control are altered with (mindfulness) meditation. There is strong evidence of positive overall effects of MBIs in children as well as adults $[21,149,150]$. However, studies investigating the effects of MBIs on self-control in youth with ADHD and/or their parents are scarce and there is a need for methodologically stronger trials.

This protocol describes an adequately powered RCT studying family $\mathrm{MBI}$ as an innovative non-pharmacological approach in the treatment of youth with ADHD. Children with comorbidities (e.g. ASD, ODD, dyslexia) will be included which has the advantage of increasing the representativeness of the sample for the clinical ADHD population. Where previous studies were uncontrolled or waitlist controlled, the current trial allows comparison with a CAU control group. Actually received CAU prior to and during study participation will be registered in both the control and intervention condition. The intervention is a manualised family MBI (MYmind) for children and their parents given by well trained experienced mindfulness teachers who will be evaluated in terms of both their adherence to the protocol and competence. Another strength of the study is that assessments will be done with different informants allowing taking account of rater effects. Questionnaires will not only be rated by self and parents, but also by teachers who are not involved in the intervention. Next to subjective ecologically valid questionnaires, objective computerised tasks will be administered to explore effects on different aspects of self-control. Further, a broad range of 
outcomes (e.g. neuropsychological functions, clinical symptoms, positive health) will be assessed in both the child and the parent. This not only allows studying the effect of family MBI on child and parental outcomes but also how they relate to each other. This will increase our understanding of the influence of parental symptoms, functioning and well-being on the child and vice versa, and the possible role that MBI on a family level may play in targeting child and/ or parental needs. In addition, follow-up assessments until six months after the end of treatment make it possible to investigate both the short- and long-term effectiveness of the intervention. In a similar trial of an MBI in adults with ADHD, significant effects on self-control assessed with the BRIEF were only found at follow-up [58], as it might take more time and practice before MBI results in improvement of real-life self-control skills. Effects of self-control are suggested to follow a continuum, therefore interventions that achieve even small improvements in self-control for individuals, could shift outcomes across the population as a whole in a positive direction to impact health, wealth and crime rates [1]. Finally, ADHD is one of several psychopathologies (e.g. ODD, CD, addictions, mood- and anxiety disorders) that involve self-control deficits. Hence, results of the proposed RCT are in a cross-disorder perspective informative for a broad clinical population.

\section{Additional file}

Additional file 1: Neurocognitive tests instructions. Neurocognitive test instructions (translated from Dutch). (DOCX $18 \mathrm{~kb}$ )

\footnotetext{
Abbreviations

ADHD: Attention Deficit/Hyperactivity Disorder; AE: Adverse Event; AQ10: Short Autism Spectrum Quotient; ASD: Autism Spectrum Disorder: BEQC: Body Experience Questionnaire for Children; BRIEF: Behaviour Rating Inventory of Executive Function; CAMM: Child and Adolescent Mindfulness Measure; CAU: Care-As-Usual; CD: Conduct Disorder; CEQ: Credibility/ Expectancy Questionnaire; CMO: Central committee on research involving human subjects; CPRS-R:L: Conners' Parent Rating Scales-Revised: Long; CTRSR:L: Conners' Teacher Rating Scales-Revised: Long; DASS-21: Depression Anxiety Stress Scale; DSM-5: Diagnostic and Statistical Manual of Mental Disorders, Fifth Edition; DSM-IV: Diagnostic and Statistical Manual of Mental Disorders, Fourth Edition; GCP: Good Clinical Practice; HSCS: Highly Sensitive Child Scale; HSPS: Highly Sensitive Person Scale; ICU: Inventory of CallousUnemotional traits; IM-P: Interpersonal Mindfulness in Parenting scale; IQ: Intelligence Quotient; K10: Kessler Psychological Distress Scale; K-SADSPL: Schedule for Affective Disorders and Schizophrenia for School-Age Children-Present and Lifetime Version; MBCT: Mindfulness-Based Cognitive Therapy; MBI: Mindfulness-Based Intervention; MBSR: Mindfulness-Based Stress Reduction; MHC-SF: Mental Health Continuum-Short Form; MINI: MiniInternational Neuropsychiatric Interview; ODD: Oppositional Defiant Disorder; PDS: Pubertal/Physical Development Scale; RCT: Randomised Controlled Trial; RRS: Ruminative Response Scale; SAE: Serious Adverse Event; SPIRIT: Standard Protocol Items: Recommendations for Interventional Trials; SRS: Social Responsiveness Scale; SSRT: Stop Signal Reaction Time; SWAN: Strengths and Weakness of ADHD Symptoms and Normal Behaviour; TAC: Teaching Assessment Criteria; TiC-P: Trimbos and iMTA questionnaire on Costs associated Psychiatric illness; VMBN: Association of mindfulness-based teachers in the Netherlands and Flanders; WAIS-III: Wechsler Adult Intelligence Scale-Third Edition; WHO-5: World Health Organization-Five Wellbeing Index; WISC-III: Wechsler Intelligence Scale for Children-Third Edition
}

\section{Acknowledgements}

We are grateful to Karakter Child and Adolescent Psychiatry as participating centre and for additional financial support. Further, we would like to thank Hans Groenewoud for his contribution to the randomisation procedure.

\section{Sponsor}

Stichting Karakter, Reinier Postlaan 12, 6525 GC Nijmegen, The Netherlands. The sponsor and funding bodies have no role in the design of this study and will not have any role during its execution, analyses, interpretation of the data, or decision to submit results.

\section{Dissemination policy}

Communication and dissemination activities will be focused on professionals in child and adolescent psychiatry and youth mental health, on paediatricians, and organisations engaged in guidelines on the management of ADHD and other psychiatric disorders in children, on professionals involved in prevention research and working in the educational system, and media and the general public. The findings of our study will be published in peer-reviewed scientific journals. Further results from this study will be presented at national and international conferences.

\section{Funding}

MindChamp is funded by a Horizon 2020 Marie Sklodowska-Curie Innovative Training Networks grant (C.G., J.B., grant number 643051 MiND); with additional support from the Netherlands Foundation of Mental Health (C.G., J.B., grant number 2016 7057) for the part of the study that focuses on the parents with 09/09/2016 as start date.

\section{Availability of data and materials}

Study data will be available for other researchers eighteen months after finishing the study. We will give limited access to the data, that is, data can be obtained provided that the applicant will submit a research plan and the researchers of the current study will be involved in the research on the shared data. Data will be anonymised and accompanied by the present study protocol and data list description. Other researchers can contact Karakter (sponsor of the study) to obtain data.

\section{Authors' contributions}

$A S, C G, J B, N S$ and $S B$ contributed to the design of the study. CG is principal investigator and project coordinator of the study and JB, AS and SB are co-principal investigators. NS drafted the paper, which was modified and supplemented by all other authors. NS, NW, CG and LB are involved in recruiting participants and data collection. NS, NW and CG take care of the logistics of the study. SB contributes to the training and supervision of the mindfulness teachers. All authors read and approved the final manuscript.

\section{Ethics approval and consent to participate}

This study has been ethically approved by CMO Arnhem-Nijmegen and is registered under number 2015-1938. Written informed consent will be obtained from all participating families included in the study.

\section{Consent for publication}

Not applicable.

\section{Competing interests}

$\mathrm{JB}$ has been in the past 3 years a consultant to/member of advisory board of/and/or speaker for Janssen Cilag BV, Eli Lilly, Shire, Medice, and Servier. He is not an employee of any of these companies, and not a stock shareholder of any of these companies. He has no other financial or material support, including expert testimony, patents and royalties. SB is shareholder of UVA minds and UVA minds You, in which MYmind is offered to families and teacher training in MYmind to professionals. AS is the founder and clinical director of the Radboudumc Center for Mindfulness. The other authors declared that they have no competing interests.

\section{Publisher's Note}

Springer Nature remains neutral with regard to jurisdictional claims in published maps and institutional affiliations. 


\section{Author details}

'Department of Cognitive Neuroscience, Donders Institute for Brain, Cognition and Behaviour, Radboud University Medical Centre, Nijmegen, The Netherlands. ${ }^{2}$ Karakter Child and Adolescent Psychiatry, University Center, Reinier Postlaan 12, Nijmegen 6525, GC, The Netherlands. ${ }^{3}$ Research Institute of Child Development and Education, University of Amsterdam, Amsterdam, the Netherlands. ${ }^{4}$ Department of Psychiatry, Radboudumc Centre for Mindfulness, Radboud University Medical Centre, Nijmegen, The Netherlands. ${ }^{5}$ King's College London, Social, Genetic and Developmental Psychiatry, Institute of Psychiatry, Psychology and Neuroscience, London, UK.

\section{Received: 12 June 2018 Accepted: 9 July 2018} Published online: 25 July 2018

\section{References}

1. Moffitt TE, Arseneault L, Belsky D, Dickson N, Hancox RJ, Harrington H, et al. A gradient of childhood self-control predicts health, wealth, and public safety. Proc Natl Acad Sci U S A. 2011;108(7):2693-8.

2. Duckworth AL, Kern ML. A meta-analysis of the convergent validity of selfcontrol measures. J Res Pers. 2011;45(3):259-68.

3. Strayhorn JM Jr. Self-control: theory and research. J Am Acad Child Adolesc Psychiatry. 2002;41(1):7-16.

4. Tang YY, Posner MI, Rothbart MK. Meditation improves self-regulation over the life span. Ann N Y Acad Sci. 2014;1307:104-11.

5. Caspi A, Houts RM, Belsky DW, Goldman-Mellor SJ, Harrington H, Israel S, et al. The $p$ factor: one general psychopathology factor in the structure of psychiatric disorders? Clin Psycholo Sci. 2014;2(2):119-37.

6. Biederman J, Monuteaux MC, Doyle AE, Seidman LJ, Wilens TE, Ferrero F, et al. Impact of executive function deficits and attention-deficit/hyperactivity disorder (ADHD) on academic outcomes in children. J Consult Clin Psychol. 2004;72(5):757-66.

7. Thorell LB. Do delay aversion and executive function deficits make distinct contributions to the functional impact of ADHD symptoms? A study of early academic skill deficits. J Child Psychol Psychiatry. 2007:48(11):1061-70.

8. Valiente C, Eisenberg N, Spinrad TL, Haugen R, Thompson MS, Kupfer A. Effortful control and impulsivity as concurrent and longitudinal predictors of academic achievement. J Early Adolesc. 2013;33(7):946-72.

9. Pandey A, Hale D, Goddings AL, Blakemore SJ, Viner R. Systematic review of effectiveness of universal self-regulation-based interventions and their effects on distal health and social outcomes in children and adolescents: review protocol. Syst Rev. 2017;6(1):175.

10. Masicampo EJ, Baumeister RR. Relating mindfulness and self-regulatory processes. Psychol Inq. 2007;18(4):255-8.

11. Jansen P, Schulze A, Nottberg C. Influence of mindfulness-based interventions on executive functions in childhood: a systematic review. Z Entwicklungspsychol Padagog Psychol. 2016;48(4):206-16.

12. Diamond A. Activities and programs that improve Children's executive functions. Curr Dir Psychol Sci. 2012;21(5):335-41.

13. Kabat-Zinn J. Full catastrophe living : how to cope with stress, pain and illness using mindfulness meditation. London: Piatkus; 1990.

14. Kabat-Zinn J. Mindfulness-based interventions in context: past, present, and future. Clin Psychol-Sci Pr. 2003;10(2):144-56.

15. Tang $Y Y$, Holzel BK, Posner MI. The neuroscience of mindfulness meditation. Nat Rev Neurosci. 2015;16(4):213-25.

16. Fox KC, Nijeboer S, Dixon ML, Floman JL, Ellamil M, Rumak SP, et al. Is meditation associated with altered brain structure? A systematic review and meta-analysis of morphometric neuroimaging in meditation practitioners. Neurosci Biobehav Rev. 2014;43:48-73.

17. Fox KC, Dixon ML, Nijeboer S, Girn M, Floman JL, Lifshitz M, et al. Functional neuroanatomy of meditation: a review and meta-analysis of 78 functional neuroimaging investigations. Neurosci Biobehav Rev. 2016;65:208-28.

18. Tang $Y Y$, Yang L, Leve LD, Harold GT. Improving executive function and its neurobiological mechanisms through a mindfulness-based intervention: advances within the field of developmental neuroscience. Child Dev Perspect. 2012;6(4):361-6.

19. Lao SA, Kissane D, Meadows G. Cognitive effects of MBSR/MBCT: a systematic review of neuropsychological outcomes. Conscious Cogn. 2016:45:109-23.

20. Mak C, Whittingham K, Cunnington R, Boyd RN. Efficacy of mindfulnessbased interventions for attention and executive function in children and adolescents-a systematic review. Mindfulness (N Y). 2018;9(1):59-78.
21. Zoogman S, Goldberg SB, Hoyt WT, Miller L. Id, Goldberg SBOhoo. Mindfulness interventions with youth: A meta-analysis. Mindfulness (N Y). 2015;6(2):290-302.

22. Flook L, Smalley SL, Kitil MJ, Galla BM, Kaiser-Greenland S, Locke J, et al. Effects of mindful awareness practices on executive functions in elementary school children. J Appl Sch Psychol. 2010;26(1):70-95.

23. Barkley RA. ADHD and the nature of self-control. New York. London: Guilford Press; 1997

24. Polanczyk G, de Lima MS, Horta BL, Biederman J, Rohde LA. The worldwide prevalence of ADHD: a systematic review and metaregression analysis. AmJPsychiatry. 2007;164(6):942-8.

25. Pelham WE, Foster EM, Robb JA. The economic impact of attention-deficit/ hyperactivity disorder in children and adolescents. J Pediatr Psychol. 2007; 32(6):711-27.

26. Danckaerts M, Sonuga-Barke EJ, Banaschewski T, Buitelaar J, Dopfner M, Hollis C, et al. The quality of life of children with attention deficit/hyperactivity disorder: a systematic review. Eur Child Adolesc Psychiatry. 2010;19(2):83-105.

27. Peasgood T, Bhardwaj A, Biggs K, Brazier JE, Coghill D, Cooper CL, et al. The impact of ADHD on the health and well-being of ADHD children and their siblings. Eur Child Adolesc Psychiatry. 2016;25(11):1217-31.

28. Cappe E, Bolduc M, Rouge MC, Saiag MC, Delorme R. Quality of life, psychological characteristics, and adjustment in parents of children with attention-deficit/hyperactivity disorder. Qual Life Res. 2016;26(5):1283-94.

29. NICE. Atttention deficit hyperactivity disorder. The NICE guideline on diagnosis and management of ADHD in children, young people and adults. National Clinical Practice Guideline Number 72. London: The British Psychological Society, Leicester \& The Royal College of Psychiatrists; 2009.

30. NICE. Attention defificit hyperactivity disorder: diagnosis and management. London: The British Psychological Society, Leicester \& The Royal College of Psychiatrists; 2018.

31. Daley D, van der Oord S, Ferrin M, Cortese S, Danckaerts M, Doepfner M, et al. Practitioner review: current best practice in the use of parent training and other behavioural interventions in the treatment of children and adolescents with attention deficit hyperactivity disorder. J Child Psychol Psychiatry. 2017; https://doi.org/10.1111/jcpp.12825.

32. Coghill DR, Banaschewski T, Soutullo C, Cottingham MG, Zuddas A. Systematic review of quality of life and functional outcomes in randomized placebo-controlled studies of medications for attention-deficit/hyperactivity disorder. Eur Child Adolesc Psychiatry. 2017;26(11):1283-307.

33. Tamminga HG, Reneman L, Huizenga HM, Geurts HM. Effects of methylphenidate on executive functioning in attention-deficit/hyperactivity disorder across the lifespan: a meta-regression analysis. Psychol Med. 2016; 46(9):1791-807.

34. Biederman J, Faraone SV. Attention-deficit hyperactivity disorder. Lancet. 2005;366(9481):237-48

35. van der Oord S, Prins PJ, Oosterlaan J, Emmelkamp PM. Efficacy of methylphenidate, psychosocial treatments and their combination in school-aged children with ADHD: a meta-analysis. Clin Psychol Rev. 2008; 28(5):783-800.

36. Hennissen L, Bakker MJ, Banaschewski T, Carucci S, Coghill D, Danckaerts M, et al. Cardiovascular effects of stimulant and non-stimulant medication for children and adolescents with ADHD: a systematic review and meta-analysis of trials of methylphenidate. Amphetamines and Atomoxetine CNS Drugs. 2017;31(3):199-215.

37. Stuckelman ZD, Mulqueen JM, Ferracioli-Oda E, Cohen SC, Coughlin CG, Leckman JF, et al. Risk of irritability with psychostimulant treatment in children with ADHD: a meta-analysis. J Clin Psychiatry. 2017;78(6):e648-55.

38. Kidwell KM, van Dyk TR, Lundahl A, Nelson TD. Stimulant medications and sleep for youth with ADHD: a meta-analysis. Pediatrics. 2015;136(6):1144-53.

39. Daley D, van der Oord S, Ferrin M, Danckaerts M, Doepfner M, Cortese $\mathrm{S}$, et al. Behavioral interventions in attention-deficit/hyperactivity disorder: a meta-analysis of randomized controlled trials across multiple outcome domains. J Am Acad Child Adolesc Psychiatry. 2014; 53(8):835-47. 847.e831-835

40. Rapport MD, Orban SA, Kofler MJ, Friedman LM. Do programs designed to train working memory, other executive functions, and attention benefit children with ADHD? A meta-analytic review of cognitive, academic, and behavioral outcomes. Clin Psychol Rev. 2013;33(8):1237-52.

41. Chronis-Tuscano A, Wang CH, Woods KE, Strickland J, Stein MA. Parent ADHD and evidence-based treatment for their children: review and directions for future research. J Abnorm Child Psychol. 2017:45(3): $501-17$. 
42. Faraone SV, Asherson P, Banaschewski T, Biederman J, Buitelaar JK, RamosQuiroga JA, et al. Attention-deficit/hyperactivity disorder. Nat Rev Dis Primers. 2015;1:15020.

43. Greven CU, Asherson P, Rijsdijk FV, Plomin R. A longitudinal twin study on the association between inattentive and hyperactive-impulsive ADHD symptoms. J Abnorm Child Psychol. 2011;39(5):623-32.

44. Langley K, Fowler T, Ford T, Thapar AK, van den Bree M, Harold G, et al. Adolescent clinical outcomes for young people with attention-deficit hyperactivity disorder. Br J Psychiatry. 2010;196(3):235-40.

45. Swanson JM, Arnold LE, Molina BSG, Sibley MH, Hechtman LT, Hinshaw $\mathrm{SP}$, et al. Young adult outcomes in the follow-up of the multimodal treatment study of attention-deficit/hyperactivity disorder: symptom persistence, source discrepancy, and height suppression. J Child Psychol Psychiatry. 2017:58(6):663-78.

46. Valero M, Cebolla A. Mindfulness training for adults and children with ADHD: variables and outcomes. Current Developmental Disorders Reports. 2017:4(3):95-9.

47. Modesto-Lowe V, Chaplin M, Godsay V, Soovajian V. Parenting teens with attention-deficit/hyperactivity disorder: challenges and opportunities. Clin Pediatr (Phila). 2014;53(10):943-8.

48. Cassone AR. Mindfulness training as an adjunct to evidence-based treatment for ADHD within families. J Atten Disord. 2015;19(2):147-57.

49. Rayan A, Ahmad M. Mindfulness and parenting distress among parents of children with disabilities: a literature review. Perspect Psychiatr Care. 2017;54(2):324-30

50. Cairncross M, Miller CJ. The effectiveness of mindfulness-based therapies for ADHD: a meta-analytic review. J Atten Disord. 2016; https://doi.org/ 10.1177/1087054715625301

51. Evans S, Ling M, Hill B, Rinehart N, Austin D, Sciberras E. Systematic review of meditation-based interventions for children with $A D H D$. Eur Child Adolesc Psychiatry. 2017;27(1):9-27.

52. Lo HHM, Wong SWL, Wong JYH, Yeung JWK, Snel E, Wong SYS. The effects of family-based mindfulness intervention on ADHD symptomology in young children and their parents: a randomized control trial. J Atten Disord. 2017; https://doi.org/10.1177/1087054717743330.

53. Zylowska L, Ackerman DL, Yang MH, Futrell JL, Horton NL, Hale TS, et al. Mindfulness meditation training in adults and adolescents with ADHD: a feasibility study. J Atten Disord. 2008;11(6):737-46.

54. Zhang D, Chan SKC, Lo HHM, Chan CYH, Chan JCY, Ting KT, et al. Mindfulness-based intervention for Chinese children with ADHD and their parents: a pilot mixed-method study. Mindfulness (N Y). 2017;8(4): 859-72.

55. van de Weijer-Bergsma E, Formsma AR, de Bruin El, Bögels SM. The effectiveness of mindfulness training on behavioral problems and attentional functioning in adolescents with ADHD. J Child Fam Stud. 2012 21(5):775-87.

56. Mitchell JT, Mclntyre EM, English JS, Dennis MF, Beckham JC, Kollins SH. A pilot trial of mindfulness meditation training for ADHD in adulthood: impact on Core symptoms, executive functioning, and emotion dysregulation. J Atten Disord. 2013; https://doi.org/10.1177/1087054713513328.

57. Hepark S, Janssen L, de Vries A, Schoenberg PL, Donders R, Kan CC, et al. The efficacy of adapted MBCT on Core symptoms and executive functioning in adults with ADHD: a preliminary randomized controlled trial. J Atten Disord. 2015; https://doi.org/10.1177/1087054715613587.

58. Janssen L, Kan CC, Carpentier PJ, Sizoo B, Hepark S, Schellekens MPJ, et al. Mindfulness-based cognitive therapy $v$. treatment as usual in adults with ADHD: a multicentre, single-blind, randomised controlled trial. Psychol Med. 2018; https://doi.org/10.1017/s0033291718000429:1-11.

59. Daly M, Delaney L, Egan M, Baumeister RF. Childhood self-control and unemployment throughout the life span: evidence from two British cohort studies. Psychol Sci. 2015;26(6):709-23.

60. American Psychiatric Association. Diagnostic and Statistical Manual of Mental Disorders vol 4th 4th ed text revWashington: American Psychiatric Association; 2000

61. American Psychiatric Association. Diagnostic and statistical manual of mental disorders. 5th ed. Arlinton: American Psychiatric Association; 2013.

62. Conners CK, Sitarenios G, Parker JD, Epstein JN. The revised Conners' parent rating scale (CPRS-R): factor structure, reliability, and criterion validity. J Abnorm Child Psychol. 1998;26(4):257-68.

63. Bögels SM. Mindfulness training for youth with ADHD, and their parents (MYmind): a guide for mental health practitioners. in press.
64. Bögels SM, Hoogstad B. Dun IV, Schutter Sd, Restifo K. Mindfulness training for adolescents with externalizing disorders and their parents. Behav Cogn Psychoth. 2008;36(2):193-209.

65. Haydicky J, Shecter C, Wiener J, Ducharme JM. Evaluation of MBCT for adolescents with ADHD and their parents: impact on individual and family functioning. J Child Fam Stud. 2015;24(1):76-94.

66. Shecter $C$. Mindfulness training for adolescents with ADHD and their families: a time-series evaluation: University of Toronto; 2013. https://tspace. library.utoronto.ca/bitstream/1807/43718/1/Shecter_Carly_M_201311_PhD_ thesis.pdf.

67. van der Oord S, Bögels SM, Peijnenburg D. The effectiveness of mindfulness training for children with $\mathrm{ADHD}$ and mindful parenting for their parents. J Child Fam Stud. 2012;21(1):139-47.

68. de Bruin El, Blom R, Smit FM, van Steensel FJ, Bögels SM. MYmind: Mindfulness training for Youngsters with autism spectrum disorders and their parents. Autism. 2014; https://doi.org/10.1177/1362361314553279.

69. Ridderinkhof A, de Bruin El, Blom R, Bögels SM. Mindfulness-Based Program for Children with Autism Spectrum Disorder and Their Parents: Direct and Long-Term Improvements. Mindfulness (N Y). 2017; https://doi.org/10.1007/ s12671-017-0815-X.

70. Mitchell JT, Zylowska L, Kollins SH. Mindfulness meditation training for attentiondeficit/hyperactivity disorder in adulthood: current empirical support, treatment overview, and future directions. Cogn Behav Pract. 2015;22(2):172-91.

71. Good practice guidelines for teaching mindfulness-based courses(2011) [https://mindfulnessteachersuk.org.uk/pdf/teacher-guidelines.pdf] . Accessed 14 May 2018

72. Kabat-Zinn J. Full catastrophe living : using the wisdom of your body and mind to face stress, pain, and illness. New York: Delacorte Press; 1990.

73. Segal ZV, Williams JMG, Teasdale JD. Mindfulness-based cognitive therapy for depression: a new approach to preventing relapse. New York: Guilford Press; 2002.

74. Crane RS, Kuyken W, Williams JM, Hastings RP, Cooper L, Fennell MJ. Competence in teaching mindfulness-based courses: concepts, development and assessment. Mindfulness (N Y). 2012;3(1):76-84.

75. Crane RS, Eames C, Kuyken W, Hastings RP, Williams JM, Bartley T, et al. Development and validation of the mindfulness-based interventions teaching assessment criteria (MBI:TAC). Assessment. 2013;20(6):681-8.

76. Landelijke Stuurgroep Multidisciplinaire Richtlijnontwikkeling in de GGZ. Multidisciplinaire Richtlijn voor diagnostiek en behandeling van ADHD bij kinderen en jeugdigen. Utrecht: Trimbos-instituut; 2005.

77. Devilly GJ, Borkovec TD. Psychometric properties of the credibility/ expectancy questionnaire. J Behav Ther Exp Psychiatry. 2000;31(2):73-86.

78. Hakkaart-van RL. Manual Trimbos/iMTA questionnaire for costs associated with psychiatric illness (in dutch). J Consult Clin Psychol. 2002;59:12-9.

79. Hakkaart-van Roijen L: Validation of the Trimbos and iMTA questionnaire on Costs associated with Psychiatric illness' (TiC-P) for measuring health care utilisation and production loss. (2018). https://www.zonmw.nl/nl/onderzoekresultaten/doelmatigheidsonderzoek/programmas/project-detail/doelmatigheid sonderzoek/validation-of-the-trimbos-and-imta-questionnaire-on-costs-associatedwith-psychiatric-illness-tic/verslagen/. Accessed 10 Apr 2018.

80. Kaufman J, Birmaher B, Brent D, Rao U, Flynn C, Moreci P, et al. Schedule for affective disorders and schizophrenia for school-age children-present and lifetime version (K-SADS-PL): initial reliability and validity data. J Am Acad Child Adolesc Psychiatry. 1997;36(7):980-8.

81. Cornelius BL, Groothoff JW, van der Klink JJ, Brouwer S. The performance of the K10, K6 and GHQ-12 to screen for present state DSM-IV disorders among disability claimants. BMC Public Health. 2013;13:128.

82. Sheehan DV, Lecrubier $Y$, Sheehan KH, Amorim $P$, Janavs J, Weiller $E$, et al. The Mini-International Neuropsychiatric Interview (M.I.N.I.): the development and validation of a structured diagnostic psychiatric interview for DSM-IV and ICD-10. J Clin Psychiatry. 1998;59(20):22-33. quiz 34-57

83. Wechsler D. WISC-III-NL. Weschler Intelligence Scale for Children derde editie NL. Handleiding en verantwoording. London: Harcourt Assessment; 2005.

84. Wechsler D. WAIS-III. Nederlandse bewerking. Afname en scoringshandleiding. Lisse: Swets Test Publishers; 2000.

85. Silverstein AB. Validity of Wisc-R short forms. J Clin Psychol. 1975;31(4):696-7.

86. Silverstein AB. 2-subtest and 4-subtest short forms of the Wechsler adult intelligence scale-revised. J Consult Clin Psychol. 1982;50(3):415-8.

87. Schmitz KE, Hovell MF, Nichols JF, Irvin VL, Keating K, Simon GM, et al. A validation study of early adolescents' pubertal self-assessments. J Early Adolescence. 2004;24(4):357-84. 
88. Gioia GA, Isquith PK, Guy SC, Kenworthy L. BRIEF executieve functies gedragsvragenlijst: Handleiding. Amsterdam: Hogrefe; 2009.

89. Sonuga-Barke EJ, Brandeis D, Cortese S, Daley D, Ferrin M, Holtmann M, et al. Nonpharmacological interventions for ADHD: systematic review and meta-analyses of randomized controlled trials of dietary and psychological treatments. Am J Psychiatry. 2013;170(3):275-89.

90. Logan GD. On the ability to inhibit thought and action: A users' guide to the stop signal paradigm. In: DDTH C, editor. Inhibitory processes in attention, memory, and language. Carr DDTH ed. San Diego: Academic Press; 1994. p. 189-239.

91. Soreni N, Crosbie J, Ickowicz A, Schachar R. Stop signal and Conners' continuous performance tasks: test--retest reliability of two inhibition measures in ADHD children. J Atten Disord. 2009:13(2):137-43.

92. Thakkar KN, Congdon E, Poldrack RA, Sabb FW, London ED, Cannon TD, et al. Women are more sensitive than men to prior trial events on the stop-signal task. Br J Psychol. 2014;105(2):254-72.

93. Critchfield TS, Kollins SH. Temporal discounting: basic research and the analysis of socially important behavior. J Appl Behav Anal. 2001;34(1):101-22.

94. Scheres A, Dijkstra M, Ainslie E, Balkan J, Reynolds B, Sonuga-Barke E, et al. Temporal and probabilistic discounting of rewards in children and adolescents: effects of age and ADHD symptoms. Neuropsychologia. 2006; 44(11):2092-103.

95. Scheres A, Tontsch C, Thoeny AL, Kaczkurkin A. Temporal reward discounting in attention-deficit/hyperactivity disorder: the contribution of symptom domains, reward magnitude, and session length. Biol Psychiatry. 2010;67(7):641-8.

96. Bradley MM, Lang PJ. Measuring emotion: the self-assessment manikin and the semantic differential. J Behav Ther Exp Psychiatry. 1994;25(1):49-59.

97. Scheres A, de Water E, Mies GW. The neural correlates of temporal reward discounting. Wiley Interdiscip Rev Cogn Sci. 2013;4(5):523-45.

98. Scheres A, Lee A, Sumiya M. Temporal reward discounting and ADHD: task and symptom specific effects. J Neural Transm (Vienna). 2008;115(2):221-6.

99. den Ouden HEM, Daw ND, Fernandez G, Elshout JA, Rijpkema M, Hoogman $M$, et al. Dissociable effects of dopamine and serotonin on reversal learning. Neuron. 2013;80(4):1090-100.

100. Dias R, Robbins TW, Roberts AC. Dissociation in prefrontal cortex of affective and attentional shifts. Nat New Biol. 1996;380(6569):69-72.

101. Costescu CA, Vanderborght B, David DO. Reversal learning task in children with autism Spectrum disorder: a robot-based approach. J Autism Dev Disord. 2015;45(11):3715-25.

102. Boehme R, Lorenz RC, Gleich T, Romund L, Pelz P, Golde S, et al. Reversal learning strategy in adolescence is associated with prefrontal cortex activation. Eur J Neurosci. 2017;45(1):129-37.

103. Conners CK. Conners' rating scales-revised. North Tonawanda: Multi-Health Systems; 2001

104. Constantino JN, Davis SA, Todd RD, Schindler MK, Gross MM, Brophy SL, et al. Validation of a brief quantitative measure of autistic traits: comparison of the social responsiveness scale with the autism diagnostic interview-revised. J Autism Dev Disord. 2003;33(4):427-33.

105. Roeyers H, Thys M, Druart C, De Schryver M, Schittekatte MSRS. Screeningslijst voor autismespectrumstoornissen. Nederlandstalige versie. Amsterdam: Hogrefe Uitgevers BV; 2011.

106. Constantino JN, Gruber CP. Social responsiveness scale (SRS). Los Angeles: western Psychological Services; 2005.

107. Treynor W, Gonzalez R, Nolen-Hoeksema S. Rumination reconsidered: a psychometric analysis. Cognit Ther Res. 2003;27(3):247-59.

108. Raes F, Schoofs H, Hoes D, Hermans D, van den Eede F, Franck E. Reflection and brooding as subcomponents of rumination: A revision of the Ruminative Response Scale. Gedragstherapie. 2009;42(3-4):205-214.

109. Burwell RA, Shirk SR. Subtypes of rumination in adolescence: associations between brooding, reflection, depressive symptoms, and coping. J Clin Child Adolesc Psychol. 2007;36(1):56-65.

110. Ravens-Sieberer U, Herdman M, Devine J, Otto C, Bullinger M, Rose M, et al. The European KIDSCREEN approach to measure quality of life and wellbeing in children: development, current application, and future advances. Qual Life Res. 2014;23(3):791-803.

111. Ravens-Sieberer U, Erhart M, Rajmil L, Herdman M, Auquier P, Bruil J, et al. Reliability, construct and criterion validity of the KIDSCREEN-10 score: a short measure for children and adolescents' well-being and health-related quality of life. Qual Life Res. 2010;19(10):1487-500.

112. de Bruin El, Zijlstra BJH, Bögels SM. The meaning of mindfulness in children and adolescents: further validation of the child and adolescent mindfulness measure (CAMM) in two independent samples from the Netherlands. Mindfulness (N Y). 2014;5(4):422-30.

113. Greco LA, Baer RA, Smith GT. Assessing mindfulness in children and adolescents: development and validation of the child and adolescent mindfulness measure (CAMM). Psychol Assess. 2011;23(3):606-14.

114. Scholte E, Noens I. Brief-A. Vragenlijst executieve functies voor volwassenen. Handleiding. Amsterdam: Hogreve Uitgevers; 2011.

115. Weafer J, Baggott MJ, de Wit H. Test-retest reliability of behavioral measures of impulsive choice, impulsive action, and inattention. Exp Clin Psychopharmacol. 2013;21(6):475-81

116. Reddy LF, Waltz JA, Green MF, Wynn JK, Horan WP. Probabilistic reversal learning in schizophrenia: stability of deficits and potential causal mechanisms. Schizophr Bull. 2016;42(4):942-51.

117. Hurst RM, Kepley HO, McCalla MK, Livermore MK. Internal consistency and discriminant validity of a delay-discounting task with an adult self-reported ADHD sample. J Atten Disord. 2011;15(5):412-22.

118. Kooij JJ, Buitelaar JK, van den Oord EJ, Furer JW, Rijnders CA, Hodiamont PP. Internal and external validity of attention-deficit hyperactivity disorder in a population-based sample of adults. Psychol Med. 2005;35(6):817-27.

119. Hoekstra RA, Bartels M, Cath DC, Boomsma DI. Factor structure, reliability and criterion validity of the autism-Spectrum quotient (AQ): a study in Dutch population and patient groups. J Autism Dev Disord. 2008;38(8):1555-66.

120. Allison C, Auyeung B, Baron-Cohen S. Toward brief "red flags" for autism screening: the short autism Spectrum quotient and the short quantitative checklist for autism in toddlers in 1,000 cases and 3,000 controls. J Am Acad Child Adolesc Psychiatry. 2012;51(3):338.

121. Henry JD, Crawford JR. The short-form version of the depression anxiety stress scales (DASS-21): construct validity and normative data in a large nonclinical sample. Br J Clin Psychol. 2005;44(2):227-39.

122. de Beurs E, Van Dyck R, Marquenie LA, Lange A, Blonk RWB. De DASS: een vragenlijst voor het meten van depressie, angst en stress. Gedragstherapie. 2001;34:35-53.

123. Folker $\mathrm{H}$, Folker AP. WHO-5 as a simple method for measuring quality of life in daily psychiatric clinics. Ugeskr Laeger. 2008;170(10):830-4.

124. Topp CW, Ostergaard SD, Sondergaard S, Bech P. The WHO-5 well-being index a systematic review of the literature. Psychother Psychosom. 2015;84(3):167-76.

125. Lamers SM, Westerhof GJ, Bohlmeijer ET, ten Klooster PM, Keyes CL. Evaluating the psychometric properties of the mental health continuumshort form (MHC-SF). J Clin Psychol. 2011;67(1):99-110,

126. Bruin El, De Zijlstra BJ, Geurtzen N, van Zundert RM, van de Weijer-Bergsma E, Hartman EE, et al. Mindful parenting assessed further: psychometric properties of the Dutch version of the interpersonal mindfulness in parenting scale (IM-P). Mindfulness (N Y). 2014;5(2):200-12.

127. Duncan LG, Coatsworth JD, Greenberg MT. A model of mindful parenting: implications for parent-child relationships and prevention research. Clin Child Fam Psychol Rev. 2009;12(3):255-70.

128. Emck C, Plouvier M. Van der lee-Snel M. Body experience in children with intellectual disabilities with and without externalising disorders. Body, movement and dance in. Psychotherapy. 2012;7(4):263-75.

129. Pluess M, Assary E, Lionetti F, Lester K, Krapohl E, Aron EN, et al. Environmental sensitivity in children: development of the highly sensitive child scale and identification of sensitivity groups. Dev Psychol. 2018;54(1):51-70.

130. Herpers PCM, Klip H, Rommelse NNJ, Taylor MJ, Greven CU, Buitelaar JK. Taxometric analyses and predictive accuracy of callous-unemotional traits regarding quality of life and behavior problems in non-conduct disorder diagnoses. Psychiatry Res. 2017;253:351-9.

131. Neff KD. The development and validation of a scale to measure selfcompassion. Self Identity. 2003;2(3):223-50.

132. Raes F, Pommier E, Neff KD, Van Gucht D. Construction and factorial validation of a short form of the self-compassion scale. Clin Psychol Psychother. 2011:18(3):250-5.

133. Swanson JM, Schuck S, Porter MM, Carlson C, Hartman CA, Sergeant JA, et al. Categorical and dimensional definitions and evaluations of symptoms of ADHD: history of the SNAP and the SWAN rating scales. Int J Educ Psychol Assess. 2012;10(1):51-70.

134. Veenman B, Luman $M$, Hoeksma J, Pieterse $K$, Oosterlaan J. A randomized effectiveness trial of a behavioral teacher program targeting ADHD symptoms. J Atten Disord. 2016; https://doi.org/10.1177/1087054716658124.

135. Greven CU, Buitelaar JK, Salum GA. From positive psychology to psychopathology: the continuum of attention-deficit hyperactivity disorder. J Child Psychol Psychiatry. 2018;59(3):203-12. 
136. Smolewska KA, McCabe SB, Woody EZ. A psychometric evaluation of the highly sensitive person scale: the components of sensory-processing sensitivity and their relation to the BIS/BAS and "big five". Pers Individ Dif. 2006;40(6):1269-79.

137. Brus BT, Voeten MJM. Een-Minuut-Test versie A en B. Verantwoording en handleiding. Amsterdam: Hartcourt Test Publishers; 2006.

138. Walker D, Myrick F. Grounded theory: an exploration of process and procedure. Qual Health Res. 2006;16(4):547-59.

139. Giacomini MK, Cook DJ. Users' guides to the medical literature: XXIII. Qualitative research in health care a. Are the results of the study valid? Evidence-based medicine working group. JAMA. 2000;284(3):357-62.

140. Stange JP, Eisner LR, Holzel BK, Peckham AD, Dougherty DD, Rauch SL, et al. Mindfulness-based cognitive therapy for bipolar disorder: effects on cognitive functioning. J Psychiatr Pract. 2011;17(6):410-9.

141. Borm GF, Fransen J, Lemmens WA. A simple sample size formula for analysis of covariance in randomized clinical trials. J Clin Epidemiol. 2007; 60(12):1234-8.

142. Huizinga M, Smidts DP. Age-related changes in executive function: a normative study with the Dutch version of the behavior rating inventory of executive function (BRIEF). Child Neuropsychol. 2010;17(1):51-66.

143. Han BG, Enas NH, McEntegart D. Randomization by minimization for unbalanced treatment allocation. Stat Med. 2009;28(27):3329-46.

144. Ciwit BV: Castor Electronic Data Capture. In. Amsterdam; (2016). http://castoredc.com. Accessed 14 May 2018

145. Alshurafa M, Briel M, Akl EA, Haines T, Moayyedi P, Gentles SJ, et al. Inconsistent definitions for intention-to-treat in relation to missing outcome data: systematic review of the methods literature. PLoS One. 2012;7(11):e49163.

146. Nederlandse Federatie van Universitair Medische Centra. Kwaliteitsborging mensgebonden onderzoek 2.0. Houten: Drukkerij Badoux bv; 2012.

147. Moffitt TE, Poulton R, Caspi A. Lifelong impact of early self-control. Childhood Self-Discipline Predicts Adult Quality of Life Am Sci. 2013;101(5):352-9.

148. Erskine HE, Norman RE, Ferrari AJ, Chan GC, Copeland WE, Whiteford HA, et al. Long-term outcomes of attention-deficit/hyperactivity disorder and conduct disorder: a systematic review and meta-analysis. J Am Acad Child Adolesc Psychiatry. 2016;55(10):841-50.

149. Klingbeil DA, Renshaw TL, Willenbrink JB, Copek RA, Chan KT, Haddock A, et al. Mindfulness-based interventions with youth: a comprehensive metaanalysis of group-design studies. J Sch Psychol. 2017;63:77-103.

150. Goldberg SB, Tucker RP, Greene PA, Davidson RJ, Wampold BE, Kearney DJ, et al. Mindfulness-based interventions for psychiatric disorders: a systematic review and meta-analysis. Clin Psychol Rev. 2018;59:52-60.

Ready to submit your research? Choose BMC and benefit from:

- fast, convenient online submission

- thorough peer review by experienced researchers in your field

- rapid publication on acceptance

- support for research data, including large and complex data types

- gold Open Access which fosters wider collaboration and increased citations

- maximum visibility for your research: over $100 \mathrm{M}$ website views per year

At $\mathrm{BMC}$, research is always in progress.

Learn more biomedcentral.com/submissions 